\title{
Efficient smoothed particle radiation hydrodynamics II: Radiation hydrodynamics
}

\author{
Brody R. Bassett ${ }^{a, \star}$, J. Michael Owen ${ }^{a}$, Thomas A. Brunner ${ }^{a}$ \\ bassett4@llnl.gov, owen8@llnl.gov, brunner6@llnl.gov \\ ${ }^{a}$ Lawrence Livermore National Laboratory \\ 7000 East Avenue, Livermore, CA, 94550 \\ ${ }^{\star}$ Corresponding author
}

\begin{abstract}
The radiation hydrodynamics equations for smoothed particle hydrodynamics are derived by operator splitting the radiation and hydrodynamics terms, including necessary terms for material motion, and discretizing each of the sets of equations separately in time and space. The implicit radiative transfer discussed in the first paper of this series is coupled to explicit smoothed particle hydrodynamics. The result is a multi-material meshless radiation hydrodynamics code with arbitrary opacities and equations of state that performs well for problems with significant material motion. The code converges with second-order accuracy in space and first-order accuracy in time to the semianalytic solution for the Lowrie radiative shock problem and has competitive performance compared to a mesh-based radiation hydrodynamics code for a multi-material problem in two dimensions and an ablation problem inspired by inertial confinement fusion in two and three dimensions.
\end{abstract}

Keywords: radiation hydrodynamics, smoothed particle hydrodynamics, radiative transfer, meshless method 


\section{Introduction}

Radiation hydrodynamics adds important physics to the standard hydrodynamics equations, including energy transfer by radiative processes and radiation effects that alter the hydrodynamics equations directly. Radiation typically transfers energy at speeds that far exceed those of fluid flow or thermal conduction, and can noticeably affect the hydrodynamic system at temperatures of thousands of degrees Kelvin. Due to the dependence of radiation energy on the fourth power of the temperature, when the temperature increases a few orders of magnitude beyond that, the radiation additionally imparts significant momentum and pressure to the fluid [1]. The coupled radiation hydrodynamic equations are necessary in order to study a wide variety of problems in astrophysics such as star formation, stellar structure and evolution, accretion around stellar and collapsed objects (such as neutron stars, white dwarves, and black holes), supernova, active galactic nuclei, galaxy formation, etc. Radiation hydrodynamics is also important for high-energy density experiments, such as inertial confinement fusion research [2,3]. In this paper radiation hydrodynamics is numerically modeled with the assumption that the radiative and material energies are significantly (but non-trivially) coupled, i.e. when simplifying assumptions such as the matter is either decoupled from the radiation (free-streaming) or so tightly bound that the photons are simply another component of the fluid and can be accounted for by a modified equation of state are not appropriate.

Smoothed particle hydrodynamics (SPH) is a meshfree method typically formulated for hydrodynamics only, i.e., without including the effects of radiation. In the first paper of this series (hereafter Paper I) an efficient flux-limited diffusion treatment for radiation (coupled to the material energy) is derived based upon an SPH differencing operator, which is therefore both meshfree and well-suited to combine with SPH to create a meshfree radiation hydrodynamic method. Several implementations of radiation within SPH are discussed in Paper I, including ray tracing [4] and Monte Carlo methods [5]. Flux-limited diffusion, the approximation used here, remains the most common method due to its simplicity. Early efforts using ideal gas laws and simple, iterative convergence schemes $[6,7,8]$, which were later generalized to more difficult equations of state and more robust solution methods [9], including the similar optically-thin Variable Eddington Factor method [10]. Many of the problems that have been tested in SPH flux-limited diffusion are in 1D, and they do not always have a known solution. The 2D and 3D problems that have been studied are mostly astrophysical problems such as star formation without a reference solution, although in at least one case a code-to-code comparison is used to check the solution [10].

The general equations of radiation hydrodynamics contain relativistic terms due to the different reference frames of the fluid and radiation. Approximations on the order of the fluid velocity over the speed of light are generally applied to simplify the equations for problems with nonrelativistic fluid velocities $[11,1,12,13,14]$. The radiation transport equation can then be integrated over angle to produce the moment equations. The diffusion equations presented in Ref. [1] are those used in this paper. Similar equations could be derived from the angularly-dependent transport equation in Ref. [12] or [14]. While this paper assumes a simple relativistic approximations for the radiation equations, there are more detailed treatments tailored to diffusion that may provide additional accuracy in certain regimes [15]. Radiation hydrodynamics has long been studied in the context of meshed methods (such as Eulerian, Lagrangian, AMR, and ALE methods; see the extensive discussion in [1]), but comparatively little work has been done on meshfree methods such the one here.

In Paper I, solution methods for the coupled radiation diffusion and material energy equations for SPH are derived and verified. In Sec. 2 of this paper, the equations of radiation hydrodynamics are introduced and applied to SPH, including a fast and robust time discretization scheme that limits the number of iterations needed to converge the system. Sec. 3 briefly discusses the implementation of the methods described in Paper I and Sec. 3, which allows for the simulation of large systems relatively quickly on distributed architectures. In Sec. 4 multiple tests of the coupled radiation hydrodynamics method are presented, including a radiation hydrodynamics shock problem with a semi-analytic solution for comparison, as well as several multidimensional examples where the results are compared against a meshed method, including a problem with multiple materials and an ablation problem inspired by inertial confinement fusion.

\section{Theory}

In this section, the radiation hydrodynamics equations are introduced and discretized. The radiation hydrodynamics equations are kept general to permit an arbitrary equation of state. The equations are operator split to separate the thermal radiative transfer equations from the hydrodynamics equations, which isolates the radiation solve. The 
hydrodynamics equations are discretized explicitly in time, while the radiation equations are discretized implicitly in time. Finally, SPH spatial differencing is applied to the equations.

\subsection{The radiation hydrodynamics equations}

The radiation hydrodynamics equations are

$$
\begin{gathered}
\frac{D \rho}{D t}=-\rho \partial_{x}^{\alpha} v^{\alpha} \\
\rho \frac{D v^{\alpha}}{D t}=-\partial_{x}^{\alpha} p+\frac{\sigma_{t}}{c} F^{\alpha}, \\
\frac{D e}{D t}=-\partial_{x}^{\alpha} p v^{\alpha}-c \sigma_{a} B+c \sigma_{a} E+Q_{e}, \\
\frac{D E}{D t}=-E \partial_{x}^{\alpha} v^{\alpha}-P^{\alpha \beta} \partial_{x}^{\alpha} v^{\beta}-\partial_{x}^{\alpha} F^{\alpha}-c \sigma_{a} E+c \sigma_{a} B+Q_{E}, \\
\frac{1}{c} \frac{D F^{\alpha}}{D t}=-\frac{1}{c} F^{\alpha} \partial_{x}^{\beta} v^{\beta}-c \partial_{x}^{\beta} P^{\alpha \beta}-\sigma_{t} F^{\alpha},
\end{gathered}
$$

with the variables

$$
\begin{array}{ll}
t, & \text { time, } \\
x, & \text { position, } \\
v^{\alpha}, & \text { velocity, } \\
\rho, & \text { mass density, } \\
p, & \text { pressure, } \\
e & \text { specific material energy, } \\
E, & \text { radiation energy density, } \\
F^{\alpha}, & \text { radiation flux, } \\
P^{\alpha \beta}, & \text { radiation pressure, } \\
T, & \text { material temperature, } \\
B, & \text { integrated photon emission function, } \\
c, & \text { speed of light in a vacuum, } \\
\sigma_{t}, & \text { total opacity, } \\
\sigma_{a}, & \text { absorption opacity, } \\
a, & \text { black-body constant, } \\
Q_{e}, & \text { nonhomogeneous material energy source, } \\
Q_{E}, & \text { nonhomogeneous radiation energy source, }
\end{array}
$$

and the Lagrangian (or material) derivative,

$$
\frac{D}{D t}=\partial_{t}+v^{\alpha} \partial_{x}^{\alpha} .
$$

As in Part I, Greek letters used as superscripts indicate dimensional components of a vector and repeated indices indicate summation. 
The same energy and angular approximations apply as in Part I, namely that the transport equation has been integrated over all energy frequencies and over angle to produce the first two moments. The emission function integrated over energy is

$$
B=a T^{4} .
$$

To derive the diffusion equation, the time derivative terms in Eq. (1e) are neglected, which results in Fick's Law,

$$
F^{\alpha}=-\frac{c}{3 \sigma_{t}} \partial_{x}^{\alpha} E .
$$

A flux limiter $\lambda$ is applied to prevent the radiation from propagating faster than is physical (see Paper I for details). When these two approximations are applied to Eq. (1d), the result in the flux-limited diffusion equation,

$$
\frac{D E}{D t}=-\frac{4}{3} E \partial_{x}^{\alpha} v^{\alpha}+\partial_{x}^{\alpha}\left(\frac{c \lambda}{\sigma_{t}} \partial_{x}^{\alpha} E\right)-c \sigma_{a} E+c \sigma_{a} B+Q_{E} .
$$

The same approximations are applied to the radiation momentum term [Eq. (1b)],

$$
\rho \frac{D v^{\alpha}}{D t}=-\partial_{x}^{\alpha} p-\lambda \partial_{x}^{\alpha} E .
$$

The flux limiter in this equation prevents the radiation pressure from unphysically exceeding $E$.

\subsection{Time discretization}

Two separate operator split procedures are performed on the coupled radiation hydrodynamics equations to make their solution more tractable. These include splitting the work term from the diffusion equation and splitting the hydrodynamics from the radiative transfer, which also has the effect of splitting the Lagrangian time derivative for the radiative transfer equations. The time discretization is done separately for the hydrodynamics equations, the radiation work equation, and the TRT equations. The hydrodynamics time update is performed explicitly. The radiation work update is performed based on the explicit hydrodynamics update. Finally, the thermal radiative transfer update is performed implicitly.

\subsubsection{Radiation work}

The zeroth-moment equation [Eq. (1d)] with the Lagrangian derivative contains velocity-related terms that are required to be accurate to order $v / c$ with material motion [1],

$$
\partial_{x}^{\alpha} v^{\alpha} E+P^{\alpha \beta} \partial_{x}^{\alpha} v^{\beta} .
$$

The term $v^{\alpha} \partial_{x}^{\alpha} E$, which is included in the Lagrangian derivative, serves to entrain the radiation with the fluid flow. The other terms, $E \partial_{x}^{\alpha} v^{\alpha}+P^{\alpha \beta} \partial_{x}^{\alpha} v^{\beta}$, account for the work done in compression or expansion of the radiation energy density. For the diffusion equation, including the time derivative, this term is simplified to

$$
\frac{D E}{D t}+\frac{4}{3} E \partial_{x}^{\alpha} v^{\alpha}
$$

The velocity term is operator split, which produces a standard Lagrangian radiation diffusion equation,

$$
\frac{D E}{D t}=\partial_{x}^{\alpha}\left(\frac{c \lambda}{\sigma_{t}} \partial_{x}^{\alpha} E\right)-c \sigma_{a} E+c \sigma_{a} B+Q_{E},
$$

along with an equation that calculates work done on the radiation field,

$$
\frac{D E}{D t}=-\frac{4}{3} E \partial_{x}^{\alpha} v^{\alpha}
$$

Using the mass conservation equation [Eq. (1a)], the work equation can be written as

$$
\frac{1}{E} \frac{D E}{D t}=\frac{4}{3} \frac{1}{\rho} \frac{D \rho}{D t} .
$$


The radiation work equation [Eq. (11)] is discretized in time by integrating from the start $t_{n-1}$ to the end $t_{n}$ of a time step,

$$
\int_{t_{n-1}}^{t_{n}} \frac{1}{E} \frac{D E}{D t} d t=\frac{4}{3} \int_{t_{n-1}}^{t_{n}} \frac{1}{\rho} \frac{D \rho}{D t} d t
$$

which can be performed analytically,

$$
\ln E^{n}-\ln E^{n-1}=\frac{4}{3}\left(\ln \rho^{n}-\ln \rho^{n-1}\right),
$$

and simplified by exponentiation to

$$
\frac{E^{n}}{E^{n-1}}=\left(\frac{\rho^{n}}{\rho^{n-1}}\right)^{4 / 3} .
$$

This is similar to the treatment of work in Ref. [16]. After the hydrodynamics update has been performed, the ratio of the mass densities is used to update the radiation energy density before the thermal radiative transfer update.

\subsubsection{Radiation and hydrodynamics operator split}

SPH hydrodynamics is traditionally solved using explicit time steps, but explicit time steps for radiation diffusion would be cost prohibitive due to the small time step needed to resolve the speed of propagation for radiation using the Courant-Friedrichs-Lewy (CFL) condition, which limits the propagation of a signal within each time step to some fraction of the element size. To solve the hydrodynamics equations explicitly and the diffusion equations implicitly, the two must be operator split.

This second operator split produces two sets of equations, the hydrodynamics equations (including a term for the radiative momentum deposition) which are advanced explicitly in time,

$$
\begin{gathered}
\frac{D \rho}{D t}=-\rho \partial_{x}^{\alpha} v^{\alpha}, \\
\rho \frac{D v^{\alpha}}{D t}=-\partial_{x}^{\alpha} p-\lambda \partial_{x}^{\alpha} E, \\
\rho \frac{D e}{D t}=-\partial_{x}^{\alpha} p v^{\alpha}, \\
\frac{D E}{D t}=0
\end{gathered}
$$

and the coupled material and radiation energy equations which are advanced implicitly in time,

$$
\begin{gathered}
\rho \partial_{t} e=-c \sigma_{a} B+c \sigma_{a} E+Q_{e} \\
\partial_{t} E=\partial_{x}^{\alpha}\left(\frac{c \lambda}{\sigma_{t}} \partial_{x}^{\alpha} E\right)-c \sigma_{a} E+c \sigma_{a} B+Q_{E} .
\end{gathered}
$$

The hydrodynamics equations remain in the material frame of reference with Lagrangian derivatives, as needed for $\mathrm{SPH}$. The thermal radiative transfer (TRT) equations are in the lab frame of reference with Eulerian derivatives, which allows for their solution without consideration of material motion. As described in Sec. 2.2.3, the inclusion of Eq. (15d) is a formalism and does not require implementation.

\subsubsection{Hydrodynamics}

The hydrodynamics equations are discretized explicitly in time. Equation (15d), which states that the radiation energy moves along with the fluid, comes for free in a Lagrangian method if the radiation and hydrodynamics discretizations use the same points, as is the case here. During the explicit hydrodynamics update, the radiation energy density is moved along with the fluid, which satisfies Eq. (15d). This leaves a standard set of hydrodynamics equations, which is discretized using standard SPH methodology.

Each of the hydrodynamics equations [Eqs. (15)] (except for the radiation energy density equation) is written in the form

$$
\frac{D u}{D t}=g(t, u),
$$


and discretized explicitly. For example, second-order Runge-Kutta, which is used for the results in this paper, has the form

$$
\begin{gathered}
k_{1}=\Delta \operatorname{tg}\left(t_{n}, u^{n}\right), \\
k_{2}=\Delta t g\left(t_{n}+\frac{\Delta t}{2} u^{n}+\frac{1}{2} k_{1}\right), \\
u^{n+1}=u^{n}+k_{2} .
\end{gathered}
$$

The radiation momentum term $-\lambda \partial_{x}^{\alpha} E$ [Eq. (15b)] is lagged to always use the value $E^{n-1}$.

\subsubsection{Thermal radiative transfer}

The time discretization of the thermal radiative transfer equations is discussed at length in Paper I. In summary, Eqs. (16) are discretized in time using backward Euler, and solved using Newton iteration. A Schur complement is used to eliminate part of the Jacobian, which results in the radiation diffusion equation

$$
\frac{1}{\Delta t} E^{\ell+1}-\partial_{x}^{\alpha}\left(\frac{c \lambda}{\sigma_{t}} \partial_{x}^{\alpha} E^{\ell+1}\right)+c \sigma_{a} f E^{\ell+1}=\frac{1}{\Delta t} E^{n-1}+c \sigma_{a} B-(1-f) c \sigma_{a} E^{\ell}+Q_{E}^{n},
$$

where $\ell$ is the iteration index, $f$ is the Fleck factor [17],

$$
f=\left(1+c \sigma_{a} \Delta t \frac{4 a T^{3}}{\rho c_{v}}\right)^{-1}
$$

and $c_{v}$ is the specific heat. The $E$ values are all at the current time step $n$ except for $E^{n-1}$. The solution of Eq. (19a) represents a single inexact Newton iteration. The material energy equation,

$$
\frac{\rho}{\Delta t}\left(e-e^{n-1}\right)+c \sigma_{a} B=c \sigma_{a} E+Q_{e}^{n},
$$

is solved separately to convergence using a separate Newton iteration process before each diffusion solve. The solution of these equations (described in Paper I, Alg. 1) is performed after the hydrodynamics and radiation work equations have been updated. The opacities and other material parameters $\left(\sigma_{a}, f, D, \lambda, c_{v}\right.$, and $\left.\rho\right)$ are calculated based on the values from the end of the hydrodynamics update. The flux limiter, which depends on $E$, is updated after the radiation work update of $E$.

\subsubsection{Coupled system}

The time integration procedure for the coupled radiation hydrodynamics equations is presented in Alg. 1. For the hydrodynamics update, the radiation momentum term is held constant at the start-of-step value, $E^{n-1}$. Once the hydrodynamics update is complete, the radiation work is calculated. This updated radiation energy is then used in the thermal radiative transfer update. Before the radiation update, the opacities, flux limiter, specific heat, the Fleck factor are evaluated again using the updated hydrodynamics variables and the radiation energy after the work equation is applied. They are then held constant over the update, which means that the preconditioner for the diffusion solver only needs to be initialized once.

The material energy is updated twice, first during the hydrodynamics update and again during the radiation update. During the radiation update, the material energy equation [Eq. (29a)] should include the energy gained or lost over the hydrodynamics update. This is done by providing this energy as a source to the material energy equation,

$$
Q_{e}^{n}=\frac{\rho}{\Delta t} \Delta e_{\text {hydro }}^{n}=\frac{\rho}{\Delta t}\left(e_{\text {hydro }}^{n}-e^{n-1}\right) .
$$

\subsection{Spatial discretization}

The spatial discretization of the radiation hydrodynamics equations is performed using SPH, as described in Paper

I. In review, the equations are interpolated using a kernel, $W\left(x-x^{\prime}, h\right)$, with the notation

$$
\begin{aligned}
\langle g(x)\rangle & =\int_{V} W\left(x-x^{\prime}, h\right) g(x) d V^{\prime} \\
& \approx \sum_{j} V_{j} W\left(x-x_{j}, h\right) g\left(x_{j}\right) .
\end{aligned}
$$


Derivatives are approximated similarly,

$$
\begin{aligned}
\left\langle\partial_{x}^{\alpha} g(x)\right\rangle & =\int_{V} W\left(x-x^{\prime}, h\right) \partial_{x^{\prime}}^{\alpha} g\left(x^{\prime}\right) d V^{\prime} \\
& =-\int_{V} \partial_{x^{\prime}}^{\alpha} W\left(x-x^{\prime}, h\right) g\left(x^{\prime}\right) d V^{\prime} \\
& =\partial_{x}^{\alpha} \int_{V} W\left(x-x^{\prime}, h\right) g\left(x^{\prime}\right) d V^{\prime}-g(x) \partial_{x}^{\alpha} \int_{V} W\left(x-x^{\prime}, h\right) d V^{\prime} \\
& \approx \sum_{j} V_{j}\left(g_{j}-g(x)\right) \partial_{x}^{\alpha} W\left(x-x_{j}, h\right) .
\end{aligned}
$$

Note that the $g(x)$ term is zero in the continuous derivative (since $W$ is an even function), but is not necessarily zero in the discrete approximation, as the quadrature points $x_{j}$ are not always symmetric. This term ensures that the discrete derivative is zero for a constant function.

The equations without spatial derivatives are multiplied by $W\left(x_{i}-x^{\prime}, h\right)$, integrated, and then evaluated using the approximation $\langle g(x)\rangle_{i} \approx g_{i}$. For the radiation work equation, this means the interpolated value,

$$
\left\langle\frac{E^{n}}{E^{n-1}}\right\rangle_{i}=\left\langle\left(\frac{\rho^{n}}{\rho^{n-1}}\right)^{4 / 3}\right\rangle_{i},
$$

becomes a simple evaluation at point $i$,

$$
\frac{E_{i}^{n}}{E_{i}^{n-1}}=\left(\frac{\rho_{i}^{n}}{\rho_{i}^{n-1}}\right)^{4 / 3}
$$

which means the update in time can be done independently for each point.

\subsubsection{Hydrodynamics}

The spatial discretization of the hydrodynamics equations [Eqs. (15)] is similar, but includes derivatives in addition to the values evaluated at specific points. To derive the SPH form of the mass conservation equation, the original equation is multiplied by the kernel centered at point $x_{i}, W\left(x_{i}-x^{\prime}, h\right)$, and integrated,

$$
\left\langle\frac{1}{\rho\left(x^{\prime}\right)} \frac{D \rho\left(x^{\prime}\right)}{D t}\right\rangle_{i}=-\left\langle\partial_{x}^{\alpha} v^{\alpha}\left(x^{\prime}\right)\right\rangle_{i}
$$

Using the approximation $\langle g(x)\rangle_{i} \approx g_{i}$ for the time derivative term and the derivative approximation in Eq. (22) for the velocity derivative, the equation becomes

$$
\frac{1}{\rho_{i}} \frac{D \rho_{i}}{D t}=-\sum_{j} V_{j}\left(v_{j}^{\alpha}-v_{i}^{\alpha}\right) \partial_{x_{i}}^{\alpha} W\left(x_{i}-x_{j}, h\right) d V^{\prime} .
$$

Performing similar manipulations to the other hydrodynamics equations results in a standard set of SPH equations [18],

$$
\begin{gathered}
\frac{D \rho_{i}}{D t}=\rho_{i} \sum_{j} V_{j}\left(v_{i}^{\alpha}-v_{j}^{\alpha}\right) \partial_{x_{i}}^{\alpha} W_{i j}, \\
\frac{D v_{i}^{\alpha}}{D t}=-\sum_{j} m_{j}\left(\frac{p_{j}}{\rho_{j}^{2}}+\frac{p_{i}}{\rho_{i}^{2}}\right) \partial_{x_{i}}^{\alpha} W_{i j}, \\
\frac{D e_{i}}{D t}=-\sum_{j} m_{j}\left(\frac{p_{i} v_{j}^{\alpha}}{\rho_{i}^{2}}+\frac{p_{j} v_{i}^{\alpha}}{\rho_{j}^{2}}\right) \partial_{x_{i}}^{\alpha} W_{i j},
\end{gathered}
$$

where

$$
W_{i j}=W\left(x_{i}-x_{j}, h\right) .
$$

Note that for the examples shown in this paper, the mass density $\rho_{i}$ is advanced using the SPH summation form,

$$
\rho_{i}=\sum_{j} m_{j} W_{i j}
$$


rather than the continuity relation of Eq. (27a), which is only used to form the half-step estimate of the mass density during the Runge-Kutta time advancement of the mass density. Similarly the material energy relation in Eq. (27c) is only used for the half-step estimate, while the actual hydrodynamic work is evaluated using the compatible energy update described in Ref. [19].

\subsubsection{Thermal radiative transfer}

The spatial discretization of the radiation equations [Eqs. 19] is described in Paper I. The result of this discretization is the equations

$$
\begin{gathered}
\frac{\rho_{i}}{\Delta t}\left(e_{i}^{n, \ell+1}-e_{i}^{n-1}\right)+c \sigma_{a, i} B_{i}^{n, \ell+1}-c \sigma_{a, i} E_{i}^{n, \ell+1}-Q_{e, i}^{n}=0 \\
\frac{1}{\Delta t} E_{i}^{n, \ell+1}-\sum_{j} V_{j}\left(D_{i}+D_{j}\right)\left(E_{i}^{n, \ell+1}-E_{j}^{n, \ell+1}\right) \frac{x_{i j}^{\alpha}}{x_{i j}^{\beta} x_{i j}^{\beta}} \partial_{x_{i}}^{\alpha} W_{i j}+c \sigma_{a, i} f_{i} E_{i}^{n, \ell+1} \\
=\frac{1}{\Delta t} E_{i}^{n-1}+c \sigma_{a, i} B_{i}^{n, \ell}-\left(1-f_{i}\right) c \sigma_{a, i} E_{i}^{n, \ell}+Q_{E, i}^{n}
\end{gathered}
$$

with the diffusion coefficient $D$ and Fleck factor $f$ evaluated at point $i$,

$$
\begin{gathered}
D_{i}=\frac{c \lambda_{i}}{\sigma_{t, i}} \\
f_{i}=\left(1+c \sigma_{a, i} \Delta t \frac{4 a T_{i}^{3}}{\rho_{i} c_{v, i}}\right)^{-1} .
\end{gathered}
$$

The diffusion equation is solved using GMRES with the Hypre BoomerAMG preconditioner [20].

\section{Methodology}

The radiation hydrodynamics methods from this paper are implemented in the SPH code Spheral, which is available at https://github.com/jmikeowen/spheral. Note, however, that the publicly available version of Spheral does not include the radiation evolution described here and in Paper I. Please contact the authors for access to the thermal radiative transfer code and the input scripts for the problems in Sec. 4.

As in Paper I, the Wendland C4 kernel is used for the results in this paper,

$$
W_{\text {wendland }}\left(x_{i j}, h\right)= \begin{cases}k(1-r)^{5}(5 r+1), & r \equiv x_{i j} / h \leq 1, \\ 0, & \text { otherwise }\end{cases}
$$

where $k$ is a normalization constant. The standard SPH evaluation of the kernel $\psi(r)$ uses a scalar $h$,

$$
r=\frac{\sqrt{\left(x^{\alpha}-x^{\alpha, \prime}\right)\left(x^{\alpha}-x^{\alpha, \prime}\right)}}{h} .
$$

Although Spheral incorporates ASPH (a tensor generalization of the scalar smoothing scale $h$, as described in [21]), the examples in this paper simply use the scalar smoothing scale of SPH. The smoothing scale $h_{i}$ is spatially (pointwise) varying, and is adapted such that each point samples approximately a constant number of neighbors within the sampling kernel [Eq. (30)]. The algorithm for adjusting the smoothing scale per point is described in Ref [22]. Unlike in Part I, this smoothing scale is recalculated at every time step due to the movement of the SPH particles.

The code is also run with the hydrodynamics in compatible energy mode, as described in Ref. [19], which makes the hydrodynamics step exactly energy conserving. The artificial viscosity used for the problems in this paper is based on the Monaghan and Gingold viscosity [23].

\section{Results}

Three problems are considered, including a radiating shock with a semianalytic solution, a multi-material problem with strong radiation coupling, and an ablation problem. For the latter two problems, the results are compared to 
Kull, a mesh-based, arbitrary Lagrangian-Eulerian (ALE) inertial confinement fusion code at Lawrence Livermore National Laboratory [24]. All three problems use an ideal gas equation of state, with

$$
\begin{gathered}
p=(\gamma-1) \rho e, \\
T=\frac{(\gamma-1) \mu m_{p}}{k_{B}} e,
\end{gathered}
$$

where $\gamma$ is the heat capacity ratio, $\mu$ is the molecular weight, $m_{p}$ is the proton mass, and $k_{B}$ is the Boltzmann constant.

\subsection{Lowrie radiating shock}

To test the coupled radiation hydrodynamics, the planar shock wave from Lowrie and Edwards [25] is considered. The spatial profile of the solution is semianalytic and moves at a constant velocity. This problem simply translates the semianalytic solution with time across the simulated volume, so the initial conditions can be obtained from the solutions described in [25]. Two cases are considered, a Mach 2 shock with constant opacities and a Mach 45 shock with opacities that depend on density and temperature. The parameters for these shocks are listed in Table (1). The equation of state is for an ideal gas, with the atomic mass calculated from the heat capacity $c_{v}$. A radiation wave ahead of the shock, called a precursor, heats the material up to pre-shock temperatures. When the shock hits, the material is discontinuously heated up to a peak temperature, known as the Zel'dovich spike [26]. Finally, the material cools through radiative losses to post-shock temperatures. The Mach 2 case is a subcritical shock, meaning the pre-shock temperature is lower than the post-shock temperature, whereas the Mach 45 case is supercritical, meaning the pre-shock and post-shock temperatures are equal.

Ten points at the left and the right side of the problem are chosen to be boundary points with constant values of velocity, density, material energy, and radiation energy. The positions are set to move with the constant velocities assigned to the points. The smoothing parameter is fixed to its initial value for these boundary points. As the support of the kernel is less than ten points across, this mimics a constant boundary condition for the remainder of the points not on the boundary.

The solution for the Mach 2 case at the end time (after the shock has traveled $0.06 \mathrm{~cm}$ ) with 16384 total points is shown in Fig. 1a. The radiation temperature is calculated based on Eq. (3),

$$
T=\left(\frac{E}{a}\right)^{1 / 4} .
$$

The Zel'dovich spike is well-resolved, and the pre-shock and post-shock temperatures agree with the semianalytic solution. The error appears to be largest in the precursor region (around 0.2 percent $L_{\text {inf }}$ relative error), where the numerical solution appears to be more diffusive than the semianalytic solution. For the Mach 45 case, also with 16384 points, the solution after the shock has traveled $2000 \mathrm{~cm}$ is shown in Fig. 1b. The maximum relative error in this case is 0.07 percent for the material and 0.01 percent for the radiation, both of which occur near the shock front where the temperature is smallest. For the Mach 45 case, the radiation momentum term (see Sec. 2.1) is vital to calculating the solution correctly. Without radiation momentum (which has the same form as a hydrodynamic pressure), the shock lags behind the semianalytic solution, causing a density spike that changes the spatial profile of the solution (Fig. 2).

Convergence results for the Mach 2 and Mach 45 cases are shown in Figs. 3a and 3b, respectively. The numeric solution converges with second-order spatial accuracy to the semianalytic solution. For a high number of points, the error levels off due to the first-order radiation time discretization, which is chosen for stability and not accuracy. The time step for both Mach 2 and Mach 45 is limited by the CFL condition (see Sec. 2.2.3), which decreases linearly as the spacing between solution points is decreased, $\Delta t \propto \Delta x$. This couples the temporal and spatial resolution, leading to an effective first-order convergence unless the time step is manually set to lower values. To see full second-order convergence in space, the time step needs to decrease as $\Delta t \propto \Delta x^{2}$, but this becomes cost-prohibitive for large simulations. For lower numbers of points, the solutions converge with second-order accuracy despite the shock, which is because the error is largest in the precursor region.

\subsection{Triple point with radiation}

The triple point problem is traditionally a hydrodynamics-only test problem, and has been researched previously for various meshed methods such as finite element methods [27], as well as the meshfree conservative reproducing 
kernel (CRKSPH) example in [28]. The problem is changed here to start with significant radiation energy in the high-pressure side, as shown in the initial conditions in Fig. 4. The pressure of the two low-pressure regions is also decreased to allow the hydrodynamics to act on similar time scales to the radiation hydrodynamical effects. Reflecting boundary conditions are applied to each of the four boundary surfaces. The unit system for Spheral is set to agree with the fixed unit system of Kull, which is in terms of centimeters for length, grams for mass, shakes for time $\left(1 \mathrm{sh}=10^{-8} \mathrm{~s}\right)$, jerks for energy $\left(1 \mathrm{jrk}=10^{16} \mathrm{erg}\right)$, and $\mathrm{keV}$ for temperature $\left(1 \mathrm{keV}=1.16045 \times 10^{7} \mathrm{~K}\right)$. The initial conditions are also in this unit system (Fig. 4), with opacities of $\sigma_{a}=\sigma_{s}=100.0 \rho \mathrm{cm}^{-1}$ in all regions of the problem. Similarly, while the ratio of specific heats $(\gamma)$ is set per region as shown in Fig. 4, the molecular weight is set to a constant value of $\mu=1$ in all three regions.

The problem is run in Spheral and Kull in low and high resolution, with the run parameters in Table 2. In Spheral, the points are mass-matched, meaning that in the low-density region, the points have a volume that is ten times larger than in the high-density regions. Without mass matching, the boundaries between materials can become unstable and lead to unphysical instabilities. In Kull, the zones are volume-matched, meaning that the mass of the cells in the low-density region is ten times lower than the mass of the cells in the high-density regions. These differences imply there are regional resolution differences between the codes, though the number of total elements (points vs. zones) is the same in each code. The problem is run to a final time of $7.0 \mathrm{sh}$.

Figure 5 shows a comparison of the results for Spheral and Kull. The first visible feature of the problem is a radiation wave traveling in the $+\hat{x}$ direction through the upper low-density region. This causes the surface of the lower region to ablate upwards, sending an impulse in the $-\hat{y}$ direction. Meanwhile, the high-pressure region creates a shock in the $+\hat{x}$ direction, which meets the shock formed due to the ablation. At the end time, the most prominent features are the roll-up due to the Kelvin-Helmholtz instability and the trailing tails of high-density material left from the colliding shocks. For the high-resolution cases, the Spheral and Kull solutions are nearly indistinguishable. For the low-resolution cases, the arbitrary Lagrangian-Eulerian remap in Kull leads to a very diffuse result, while the limited number of particles in Spheral leads to shock boundaries that are smoothed compared to the high-resolution cases.

The difference between Kull and Spheral for the high-resolution cases, defined as

$$
\operatorname{diff}_{u}=\frac{\left|u_{\text {kull }}-u_{\text {spheral }}\right|}{\frac{1}{2}\left(u_{\text {kull }}+u_{\text {spheral }}\right)},
$$

where $u$ is the radiation energy, material energy, pressure, or density, is shown in Fig. 6. The difference is between a fraction of a percent and ten percent in most of the domain for all the state variables shown. The most visible differences are in the shock and wave timings, where the Kull radiation wave and the Kull shocks are faster than those in Spheral. This leads to large relative differences of up to 200 percent where the variables have low values in pre-shock regions. The post-shock regions agree within two percent in most cases. As the radiation energy density ranges over six orders of magnitude, small differences in the timing lead to differences that appear larger there than for the other state variables. The interfaces between the three materials are very similar in the two codes, and the main difference is in the material energy, where the timing difference between the two is most visible.

The relative difference between the initial and final energy, including kinetic, internal, and radiation, is below 0.001 for both Kull and Spheral, as shown in Table 2. The number of steps for the low-resolution cases with around 21,500 points is similar for the two codes at around 3,200. For the high-resolution case with around 2,150,000 points, Spheral took 22,244 steps and Kull took 152,843 steps, which is mostly due to deforming zones that decrease the time step though the CFL condition. The wall time per time step is about 2.5 times more in Spheral than Kull, mostly due to a higher level of connectivity in the SPH diffusion matrix that slows the computation of the multigrid preconditioner in Spheral.

\subsection{Ablation problem}

A target for inertial confinement fusion (ICF) $[2,3]$ generally consists of an outer spherical shell (or ablator) and an inner shell made from deuterium-tritium (DT) ice, which contains a low-density DT gas. A radiation source heats up the ablator, which causes it to expand outward near the surface. Due to conservation of momentum, this causes an implosion that compresses the DT ice and gas. Under appropriate conditions, this leads to fusion of the DT. For this problem, the physics of ICF are simplified to allow for comparison of the SPH discretization in Spheral with the mesh-based discretization in Kull. Unlike an actual ICF simulation, there is no radiation source outside of the initial conditions and there is no thermonuclear burn. 
The capsule is modeled with a DT gas, an inner shell of DT ice, and an outer plastic shell (CH 1\% Si) surrounded by helium gas [29]. In 2D, the shells are circles (or infinite cylinders) and the helium is bounded by a square, while in $3 \mathrm{D}$, the shells are spheres and the helium is bounded by a cube. Figure 7 shows the initial geometry in $2 \mathrm{D}$, or a slice through the geometry at $z=0$ in $3 \mathrm{D}$. The problem is run with quadrant symmetry in 2D and octant symmetry in $3 \mathrm{D}$, with reflecting boundary surfaces along the coordinate axes and at the edges of the square or cube. The unit system is identical to the one in Sec. 4.2. The initial parameters are listed in Table 3. The opacities are least squares fits of tabulated opacity data [30]. To make mass matching of the points easier, the initial densities of the DT gas and the helium are around 100 times larger than what would be expected in a standard ICF capsule. The material temperature and the radiation temperature [Eq. (33)] are set equal at the starting time. In 2D, the codes are run until $1.75 \mathrm{sh}$, while in $3 \mathrm{D}$, the codes are run until $0.7 \mathrm{sh}$.

The number of points used in each code is listed in Table 4. In Kull, the initial mesh is created with a square (2D) or cube (3D) meshed region in the center of the DT gas sphere with rounded edges, with a half-length equal to half of the radius of the DT gas region. Zones are then placed radially outward from there, with the resolution in angle fixed based on the number of regions in the square or cube. The zones are mass matched across interfaces, which leads to zones in the ice and ablator that are much smaller in the radial dimension than in the swept angular dimensions. In Spheral, the number of radial points for each region is chosen based on mass matching. The points are initialized on concentric rings, with azimuthal spacing set equal to the local radial spacing. The radial spacing is ratioed, with the ratio defined as the radial distance at the outside radius over the radial distance at the inside radius. The ratios are chosen in $2 \mathrm{D}$ such that the points have smoothing lengths that do not differ drastically at material boundaries. Spheral supports anisotropic point spacing through adaptive SPH [21, ?], which would decrease the number of points needed for a given radial resolution, but to keep these examples consistent with standard $\mathrm{SPH}$, this is not employed here.

The comparison results for $2 \mathrm{D}$ are shown in Fig. 8. At the initial time, the radiation in the helium gas heats the surface of the ablator. As the ablator heats up, its opacities decrease, letting the radiation penetrate further into the ablator. The rate at which this occurs is governed by the thermal radiative transfer equations. The ablation of the outer surface of the capsule creates an inward-moving shock. This shock travels through the DT ice and eventually compresses the DT gas. The shock travels through the DT gas and converges at the center of the problem before the DT ice has stopped traveling inward. The shock in the gas rebounds and bounces back off the ice before the inward momentum of the ice is halted and the gas reaches peak densities. The DT gas then pushes back against the DT ice, which causes Rayleigh-Taylor instabilities at the gas-ice interface.

The ablation rate of the capsule in the two codes is very similar. The difference in the position of the ablator-ice interface, divided by the initial radius of the interface, is around 0.15 percent $(0.0001 \mathrm{~cm})$ for most of the simulation, and is at most 1.5 percent $(0.001 \mathrm{~cm})$ near the end of the simulation. The ice-gas interface has similar differences until the Rayleigh-Taylor instability develops, which makes visual comparison difficult. The velocity plot shows the Spheral shocks in the gas leading by about $0.02 \mathrm{sh}$, or 1.1 percent of the total simulation time. The velocities between the codes are otherwise very similar. The thermal energy and density are also very similar, with the exception of the shape of the Rayleigh-Taylor instability near the end time, which may be seeded by asymmetric initial conditions. Table 5 shows the timing and energy balance information for each case. The computational cost for the two codes is similar, as Spheral is more expensive per time step but takes fewer time steps. The relative difference between the initial and final energies is approximately the same between the two codes, at about 0.001 for Spheral and 0.002 for Kull.

Figure 10 shows the results in 3D, which are not as well resolved as in $2 \mathrm{D}$ due to computational cost (see Table 5 ), and because of this has only a fraction of the radial zones of the 2D calculation (see Table 4). Despite having fewer points, the radial direction is more finely resolved in Kull due to anisotropic cell lengths. The results are not converged for either code, meaning that they may change significantly if the spatial resolution is increased. Because of this, the results are less similar than in 2D. Compared to $2 \mathrm{D}$, the volume of the DT gas and ice is much smaller compared to the ablator volume, which makes the implosion progress more quickly.

The ablator-ice interface position is around 5 percent $(0.003 \mathrm{~cm})$ different for the two codes throughout the simulation. The ice-gas interface differs by more, at around 5.3 percent $(0.008 \mathrm{~cm})$ during the implosion and 9.3 percent $(0.014 \mathrm{~cm})$ after the gas rebounds. The stagnation points for the two simulations are similar in the outer regions. The shocks in Spheral are behind those in Kull by around $0.01 \mathrm{sh}$, or 1.4 percent of the total simulation time, but otherwise have similar behavior. Due to the comparatively low resolution of the $3 \mathrm{D}$ simulations, the gas-ice instability on shock rebound is not visible. 


\section{Conclusions and future work}

In this work a meshfree radiation hydrodynamics discretization based on smoothed particle hydrodynamics has been demonstrated. A careful splitting of the radiation hydrodynamics equations allows for the hydrodynamics to be solved using an explicit time advance and the radiation to be solved using an implicit time advance. The thermal radiative transfer equations are solved using the discretization from Paper I, while the hydrodynamics equations are solved using standard SPH methodology. This allows for fully meshless radiation hydrodynamics with arbitrary opacities and equations of state. By coupling with the efficient radiation solve from Paper I, the meshfree method has competitive performance when compared with a contemporary ALE radiation hydrodynamics code designed to study inertial confinement fusion.

The Lowrie shock problem in 1D has a semianalytic solution that allows verification of the radiation hydrodynamics equations. The numeric solution converges to the semianalytic solution with second-order accuracy for both the Mach 2 shock and the Mach 45 shock. Correct implementation of the coupling terms between radiation and hydrodynamics is essential for the Mach 45 problem, which has high radiation pressure and tests both the propagation of radiation and hydrodynamic shocks.

The triple point problem with radiation and an ablation problem based off of an ICF capsule are tested against a mesh-based code, Kull. In both cases, the main difference is minor timing differences in the shock propagation. The triple point problem tests the ablation rate of the top surface and the effects of radiation on the hydrodynamics. The meshless results for a highly-converged case are visually indistinguishable from the mesh-based code, and the difference between the two codes apart from the shock timing differences is between a fraction of a percent and 10 percent throughout the domain. For the ablation problem, the interface positions agree to within 0.15 to 1.5 percent to a percent in $2 \mathrm{D}$ and within 5.3 to 9.3 percent in 3D. For both problems, the meshless code runs with similar speed to the mesh-based code due to a smaller number of time steps required by the hydrodynamics in the meshless code.

As stated in Paper I, some improvements can be made to the diffusion discretization to ensure zeroth-order consistency, either based on the reproducing kernel particle method [31] or moving least squares particle hydrodynamics $[32,33]$. For accuracy in energy, a multigroup treatment would be needed, preferably coupled to an acceleration method such as linear multifrequency-grey acceleration [34]. Finally, other angular treatments, such as spherical harmonics or discrete ordinates transport, could be considered for problems with small opacities or where radiation is anisotropic.

\section{Acknowledgements}

The authors wish to acknowledge the assistance of Nick Gentile in deriving coupling terms for the radiation hydrodynamics equations.

This work was performed under the auspices of the U.S. Department of Energy by Lawrence Livermore National Laboratory under Contract DE-AC52-07NA27344. This document was prepared as an account of work sponsored by an agency of the United States government. Neither the United States government nor Lawrence Livermore National Security, LLC, nor any of their employees makes any warranty, expressed or implied, or assumes any legal liability or responsibility for the accuracy, completeness, or usefulness of any information, apparatus, product, or process disclosed, or represents that its use would not infringe privately owned rights. Reference herein to any specific commercial product, process, or service by trade name, trademark, manufacturer, or otherwise does not necessarily constitute or imply its endorsement, recommendation, or favoring by the United States government or Lawrence Livermore National Security, LLC. The views and opinions of authors expressed herein do not necessarily

state or reflect those of the United States government or Lawrence Livermore National Security, LLC, and shall not be used for advertising or product endorsement purposes. LLNL-JRNL-799714.

\section{References}

[1] John I Castor. Radiation hydrodynamics. Cambridge University Press, 2004.

[2] John Nuckolls, Lowell Wood, Albert Thiessen, and George Zimmerman. Laser compression of matter to superhigh densities: Thermonuclear (CTR) applications. Nature, 239(5368):139, 1972. 
[3] John Lindl. Development of the indirect-drive approach to inertial confinement fusion and the target physics basis for ignition and gain. Physics of plasmas, 2(11):3933-4024, 1995.

[4] Gabriel Altay, Rupert AC Croft, and Inti Pelupessy. Sphray: a smoothed particle hydrodynamics ray tracer for radiative transfer. Monthly Notices of the Royal Astronomical Society, 386(4):1931-1946, 2008.

[5] Sergei Nayakshin, Seung-Hoon Cha, and Alexander Hobbs. Dynamic monte carlo radiation transfer in SPH: radiation pressure force implementation. Monthly Notices of the Royal Astronomical Society, 397(3):1314-1325, 2009 .

[6] Stuart C Whitehouse and Matthew R Bate. Smoothed particle hydrodynamics with radiative transfer in the flux-limited diffusion approximation. Monthly Notices of the Royal Astronomical Society, 353(4):1078-1094, 2004.

[7] Stuart C Whitehouse, Matthew R Bate, and Joe J Monaghan. A faster algorithm for smoothed particle hydrodynamics with radiative transfer in the flux-limited diffusion approximation. Monthly Notices of the Royal Astronomical Society, 364(4):1367-1377, 2005.

[8] Lucio Mayer, Graeme Lufkin, Thomas Quinn, and James Wadsley. Fragmentation of gravitationally unstable gaseous protoplanetary disks with radiative transfer. The Astrophysical Journal Letters, 661(1):L77, 2007.

[9] Serge Viau, Pierre Bastien, and Seung-Hoon Cha. An implicit method for radiative transfer with the diffusion approximation in smooth particle hydrodynamics. The Astrophysical Journal, 639(1):559, 2006.

[10] Margarita Petkova and Volker Springel. An implementation of radiative transfer in the cosmological simulation code gadget. Monthly Notices of the Royal Astronomical Society, 396(3):1383-1403, 2009.

[11] J Robert Buchler. Radiation transfer in the fluid frame. Journal of Quantitative Spectroscopy and Radiative Transfer, 30(5):395-407, 1983.

[12] Jim E Morel. Discrete-ordinates methods for radiative transfer in the non-relativistic stellar regime. In Computational Methods in Transport, pages 69-81. Springer, 2006.

[13] Dimitri Mihalas and Barbara Weibel Mihalas. Foundations of radiation hydrodynamics. Courier Corporation, 2013.

[14] Robert B Lowrie and Allan B Wollaber. Simple material-motion corrections for thermal radiative transport. Journal of Computational and Theoretical Transport, 43(1-7):148-161, 2014.

[15] Mark R Krumholz, Richard I Klein, Christopher F McKee, and John Bolstad. Equations and algorithms for mixed-frame flux-limited diffusion radiation hydrodynamics. The Astrophysical Journal, 667(1):626, 2007.

[16] Richard L Bowers and James Ricker Wilson. Numerical modeling in applied physics and astrophysics. Boston: Jones and Bartlett, c1991., 1991.

[17] JA Fleck Jr and JD Cummings Jr. An implicit monte carlo scheme for calculating time and frequency dependent nonlinear radiation transport. Journal of Computational Physics, 8(3):313-342, 1971.

[18] Joe J. Monaghan. Smoothed particle hydrodynamics. Reports on progress in physics, 68(8):1703, 2005.

[19] Michael J Owen. A compatibly differenced total energy conserving form of sph. International Journal for Numerical Methods in Fluids, 75(11):749-774, 2014.

[20] Robert D Falgout and Ulrike Meier Yang. hypre: A library of high performance preconditioners. In International Conference on Computational Science, pages 632-641. Springer, 2002.

[21] J Michael Owen, Jens V Villumsen, Paul R Shapiro, and Hugo Martel. Adaptive smoothed particle hydrodynamics: Methodology. ii. The Astrophysical Journal Supplement Series, 116(2):155, 1998.

[22] J Michael Owen. ASPH modeling of material damage and failure. In Proceedings of the 5th International SPHERIC Workshop, pages 297-304, Manchester, UK, Jan 2010.

[23] Joseph J Monaghan and Robert A Gingold. Shock simulation by the particle method SPH. Journal of computational physics, 52(2):374-389, 1983. 
[24] James A Rathkopf, Douglas S Miller, John M Owen, MR Zike, PG Eltgroth, NK Madsen, KP McCandless, PF Nowak, MK Nemanic, NA Gentile, et al. KULL: LLNL's ASCI inertial confinement fusion simulation code. Technical report, Lawrence Livermore National Lab., CA (US), 2000.

[25] Robert B Lowrie and Jarrod D Edwards. Radiative shock solutions with grey nonequilibrium diffusion. Shock Waves, 18(2):129-143, 2008.

[26] Yakov Borisovich Zel'dovich and Yuri Petrovich Raizer. Physics of shock waves and high-temperature hydrodynamic phenomena. Courier Corporation, 2012.

[27] Veselin A Dobrev, Truman E Ellis, Tzanio V Kolev, and Robert N Rieben. High-order curvilinear finite elements for axisymmetric lagrangian hydrodynamics. Computers \& Fluids, 83:58-69, 2013.

[28] Nicholas Frontiere, Cody D Raskin, and J Michael Owen. CRKSPH-a conservative reproducing kernel smoothed particle hydrodynamics scheme. Journal of Computational Physics, 332:160-209, 2017.

[29] Robert Tipton. An ICF test problem for single group radiation-hydrodynamics codes. Personal communication, 2016.

[30] Michael Kumbera. The opacity distribution system. Technical Report UCRL-JC-148861-ABS, Lawrence Livermore National Laboratory, 2002.

[31] Wing Kam Liu, Sukky Jun, and Yi Fei Zhang. Reproducing kernel particle methods. International journal for numerical methods in fluids, 20(8-9):1081-1106, 1995.

[32] Gary A Dilts. Moving-least-squares-particle hydrodynamics I: Consistency and stability. International Journal for Numerical Methods in Engineering, 44(8):1115-1155, 1999.

[33] Gary A Dilts. Moving least-squares particle hydrodynamics II: conservation and boundaries. International Journal for Numerical Methods in Engineering, 48(10):1503-1524, 2000.

[34] Jim E Morel, Edward W Larsen, and MK Matzen. A synthetic acceleration scheme for radiative diffusion calculations. Journal of Quantitative Spectroscopy and Radiative Transfer, 34(3):243-261, 1985. 


\begin{tabular}{|c|c|c|}
\hline & Mach 2 & Mach 45 \\
\hline \hline$c_{v}\left(\mathrm{erg} \cdot \mathrm{g}^{-1} \mathrm{~K}^{-1}\right)$ & $1.911373 * 10^{8}$ & $1.246685 \times 10^{8}$ \\
\hline$\sigma_{a}\left(\mathrm{~cm}^{-1}\right)$ & $5.773503 \times 10^{2}$ & $7.565304 \times 10^{22} \rho^{2} T^{-3.5}$ \\
\hline$\sigma_{s}\left(\mathrm{~cm}^{-1}\right)$ & 0.0 & $0.4006 \rho$ \\
\hline$\gamma($ ratio of specific heats $)$ & $5 / 3$ & $5 / 3$ \\
\hline$\mu($ molecular weight $)$ & 1.0 & 1.0 \\
\hline$\rho$ pre-shock $\left(\mathrm{g} \cdot \mathrm{cm}^{-3}\right)$ & 1.0 & 1.0 \\
\hline$\rho$ post-shock $\left(\mathrm{g} \cdot \mathrm{cm}^{-3}\right)$ & 2.286075 & 6.426142 \\
\hline$T$ pre-shock $(\mathrm{K})$ & $1.410643 \times 10^{6}$ & $1.160452 \times 10^{6}$ \\
\hline$T$ post-shock $(\mathrm{K})$ & $2.930711 \times 10^{6}$ & $8.69881 \times 10^{7}$ \\
\hline$v$ pre-shock $\left(\mathrm{cm} \cdot \mathrm{s}^{-1}\right)$ & 0.0 & 0.0 \\
\hline$v$ post-shock $\left(\mathrm{cm} \cdot \mathrm{s}^{-1}\right)$ & $-3.461705 \times 10^{7}$ & $-5.705363 \times 10^{8}$ \\
\hline End time $(\mathrm{s})$ & $1.733250 \times 10^{-9}$ & $3.505474 \times 10^{-6}$ \\
\hline Distance traveled $(\mathrm{cm})$ & 0.06 & 2000.0 \\
\hline Domain $(\mathrm{cm})$ & $0.0 \leq x \leq 0.12$ & $0.0 \leq x \leq 2500.0$ \\
\hline
\end{tabular}

Table 1: Description of the Lowrie shock problem for Mach 2 and Mach 45. For opacity evaluation, the density is in $\mathrm{g} \cdot \mathrm{cm}^{-3}$, while the temperature is in $\mathrm{K}$.

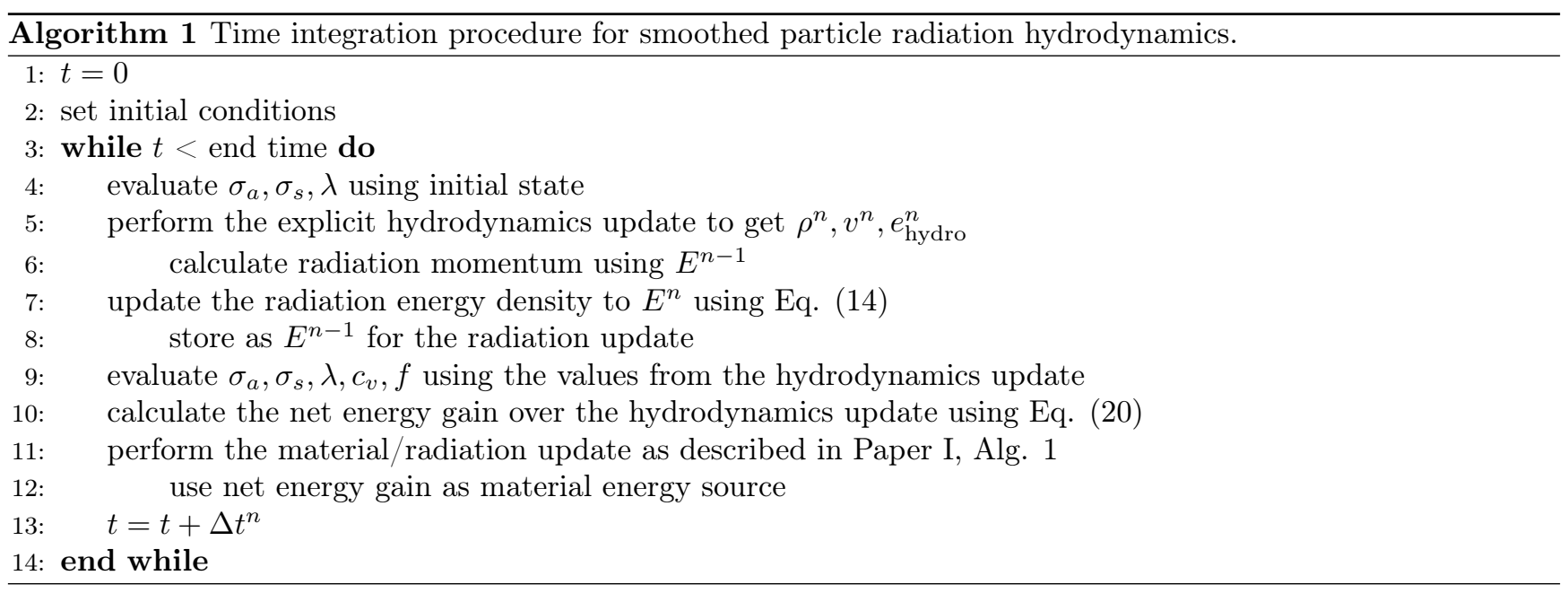




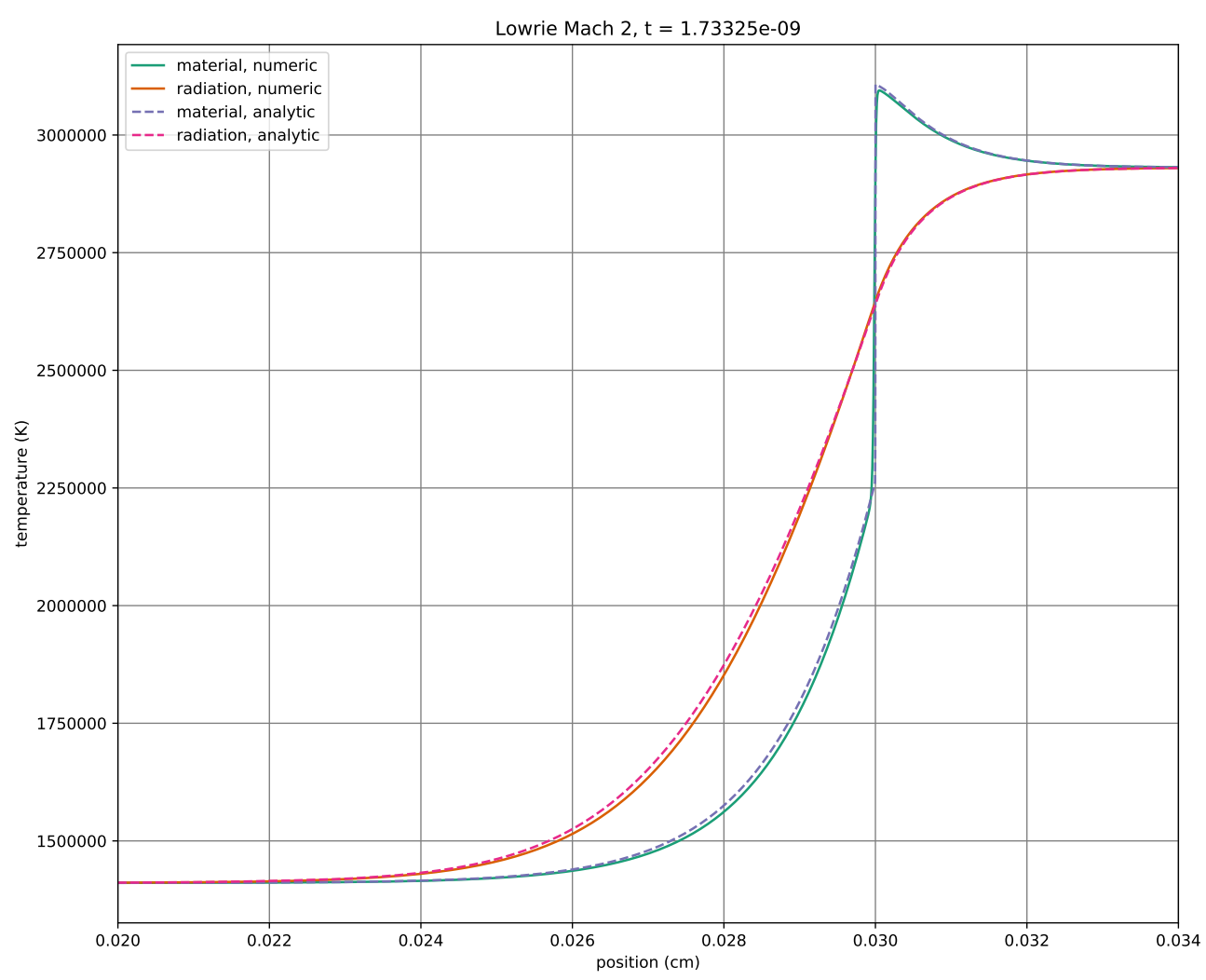

(a) Mach 2

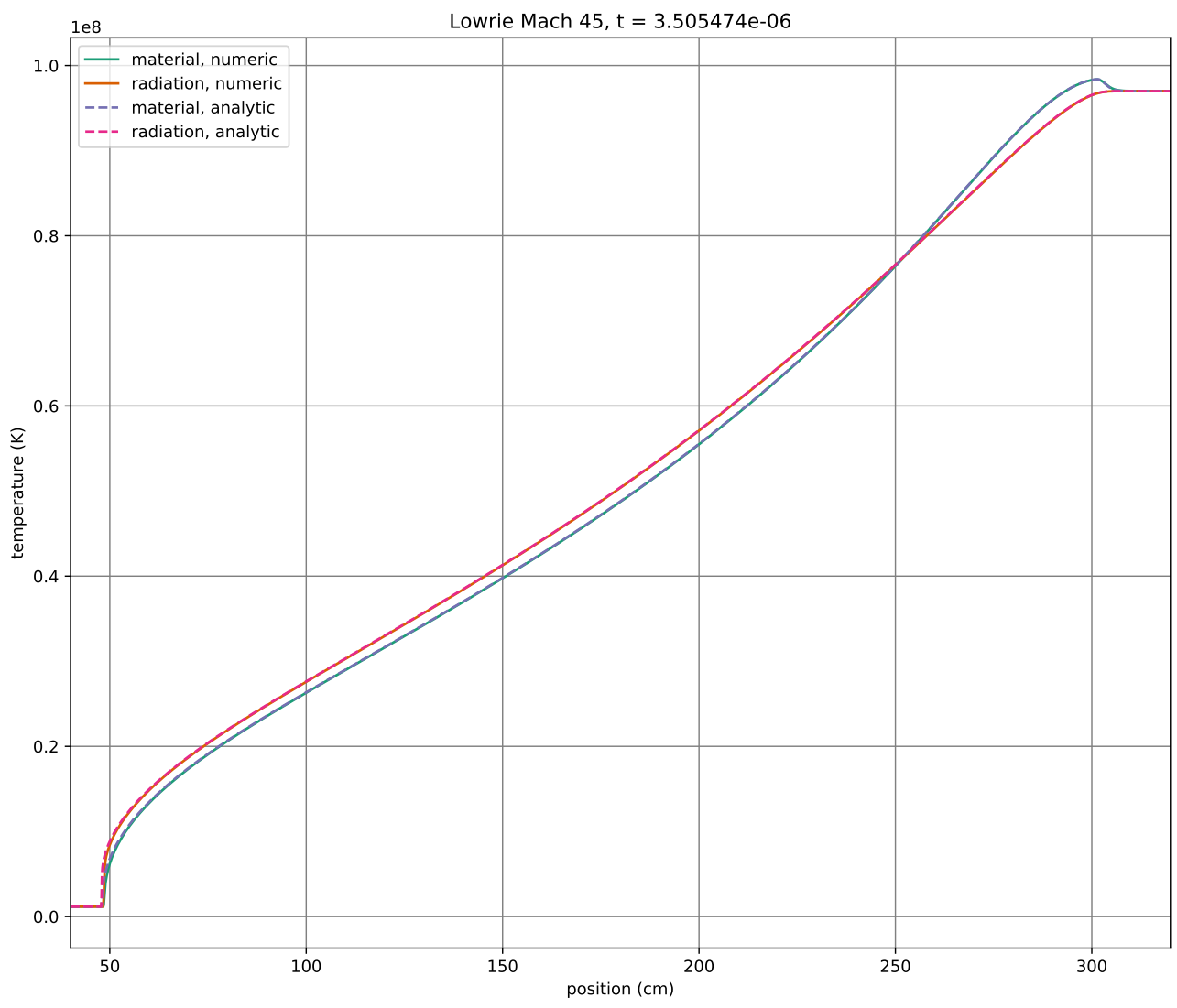

(b) Mach 45

Figure 1: Comparison of numerical and semianalytic material and radiation temperatures for the Lowrie shock, 16,384 points. 


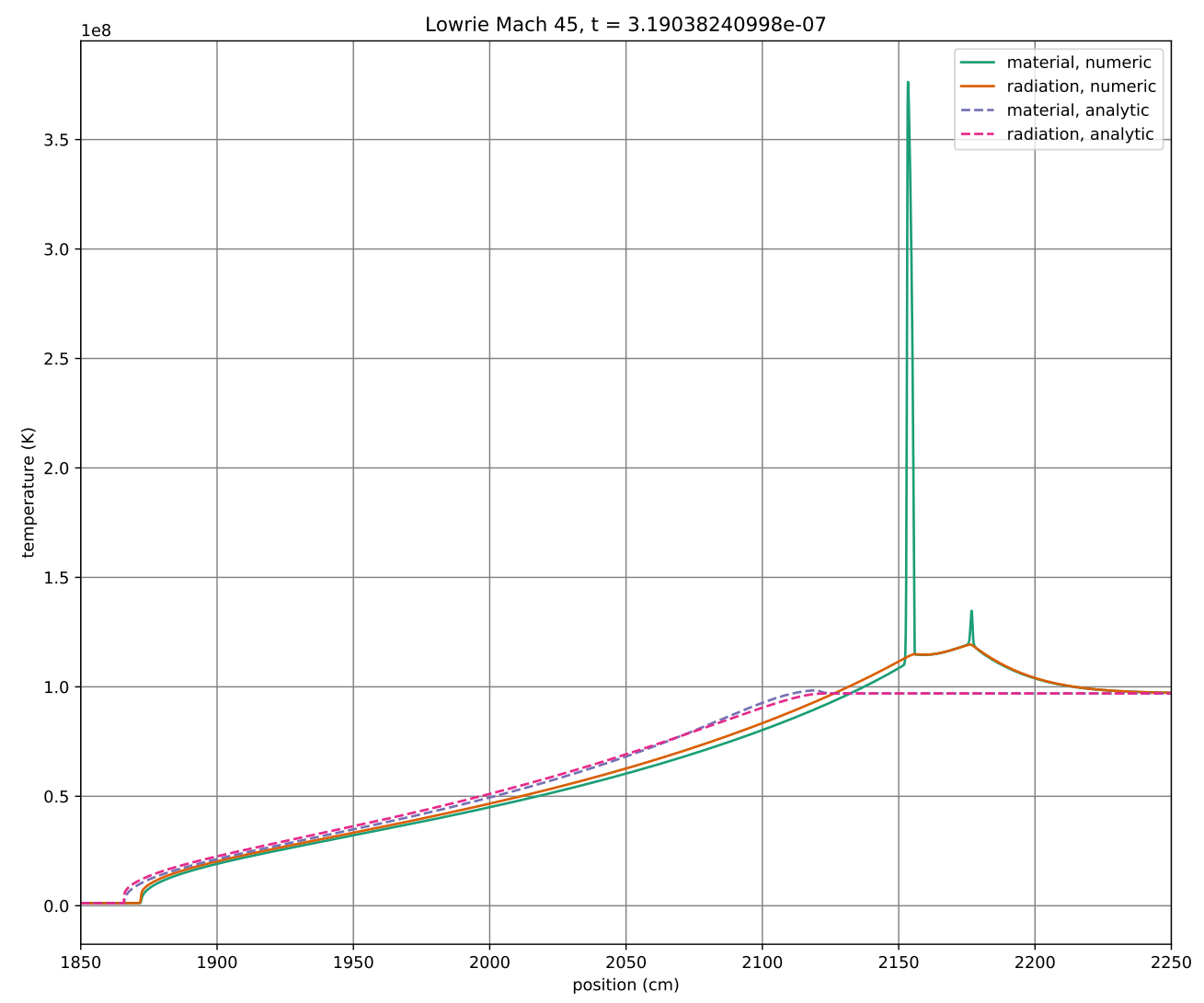

Figure 2: Comparison of numerical and semianalytic material and radiation temperatures for the Lowrie shock without radiation momentum, 16,384 points.

\begin{tabular}{|c|c|c|c|c|c|c|c|}
\hline & Points/Cells & Procs & Points/proc & Steps & Wall time & Time per step & Energy error \\
\hline \hline \multirow{2}{*}{ Spheral } & 21,410 & 36 & 595 & 3253 & 1,641 & 0.505 & $-7.466 \times 10^{-4}$ \\
\cline { 2 - 8 } & $2,149,550$ & 1152 & 1,866 & 22,244 & 62,259 & 2.799 & $-1.137 \times 10^{-4}$ \\
\hline \multirow{2}{*}{ Kull } & 21,504 & 36 & 598 & 3101 & 563 & 0.182 & $-7.449 \times 10^{-4}$ \\
\cline { 2 - 8 } & $2,150,400$ & 1152 & 1,867 & 152,843 & 170,800 & 1.117 & $2.993 \times 10^{-4}$ \\
\hline
\end{tabular}

Table 2: Run parameters and comparison of computational cost for the triple point problem. The energy error is the initial minus the final energy, divided by the initial energy.

\begin{tabular}{|c|c|c|c|c|c|c|c|}
\hline Material & Outer radius $(\mathrm{cm})$ & Initial $\rho\left(\mathrm{g} \cdot \mathrm{cm}^{-3}\right)$ & Initial $T(\mathrm{keV})$ & $\gamma$ & $\mu$ & $\sigma_{a}\left(\mathrm{~cm}^{-1}\right)$ & $\sigma_{s}\left(\mathrm{~cm}^{-1}\right)$ \\
\hline \hline DT Gas & 0.08628 & $3.0 \times 10^{-2}$ & $1.7 \times 10^{-6}$ & 1.45 & 1.0 & \multirow{2}{*}{$83.0 \rho^{1.56} T^{-2.5}$} & \\
\cline { 1 - 6 } DT Ice & 0.0943 & 0.25 & $4.2 \times 10^{-7}$ & 1.45 & 1.0 & & \multirow{2}{*}{$1.0 \rho$} \\
\cline { 1 - 6 } CH 1\% Si & 0.1108 & 1.073 & $8.6 \times 10^{-6}$ & 1.3 & 13.0 & $5.7 \rho^{1.62} T^{-2.7}$ & \\
\cline { 1 - 6 } Helium & 0.2 & $1.0 \times 10^{-2}$ & 0.4 & 1.66 & 2.0 & $0.30 \rho^{1.73} T^{-3.3}$ & \\
\hline
\end{tabular}

Table 3: Input parameters for ablation problem. For opacity evaluation, density is in $\mathrm{g} \cdot \mathrm{cm}^{-3}$ and temperature is in $\mathrm{keV}$. The listed radius for helium is the half-length of the square or cube. The $(\gamma, \mu)$ columns are the (ratio of specific heats, mean molecular weight) for the gamma-law gas equations of state in each region. 


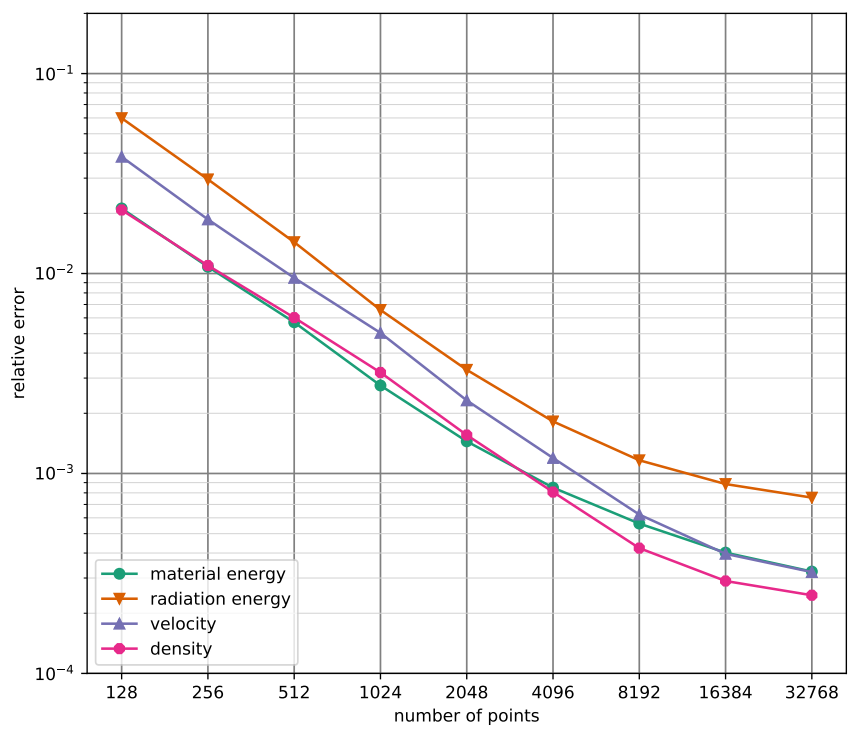

(a) Mach 2

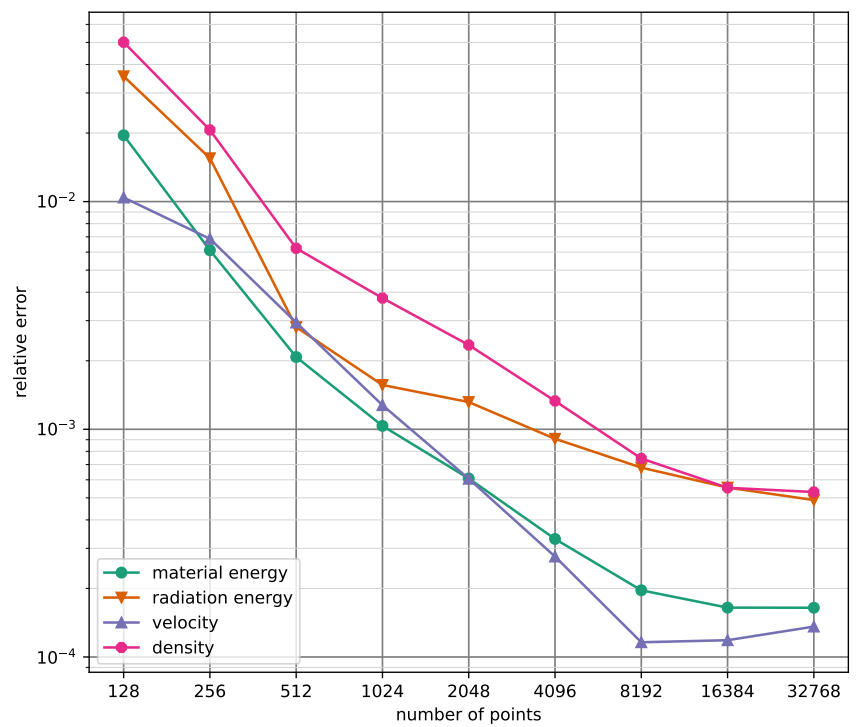

(b) Mach 45

Figure 3: Convergence of the numerical result for the Lowrie shock to the semianalytic solution.

\begin{tabular}{|c|c|c|c|c|c|c|}
\hline Code & Dimension & DT Gas & DT Ice & CH 1\% Si & Helium & Points/cells \\
\hline \hline \multirow{2}{*}{ Spheral } & 2 & $134(0.5)$ & $36(0.5)$ & $154(1.0)$ & $155(20.0)$ & 328,465 \\
\cline { 2 - 7 } & 3 & $38(1.0)$ & $7(1.0)$ & $23(1.0)$ & $52(10.0)$ & $1,058,392$ \\
\hline \multirow{2}{*}{ Kull } & 2 & 96,96 & 144 & 1296 & 128 & 328,704 \\
\cline { 2 - 7 } & 3 & 14,14 & 22 & 192 & 19 & 147,980 \\
\hline
\end{tabular}

Table 4: Number of radial points or zones in each region for the ablation problem. The numbers in parentheses for Spheral are the ratio of the radial distance between points at the inner radius to the outer radius. In the DT gas, Kull uses two parameters, the number of zones across a square or cube at the center of the region and the number of radial regions outside of it. 


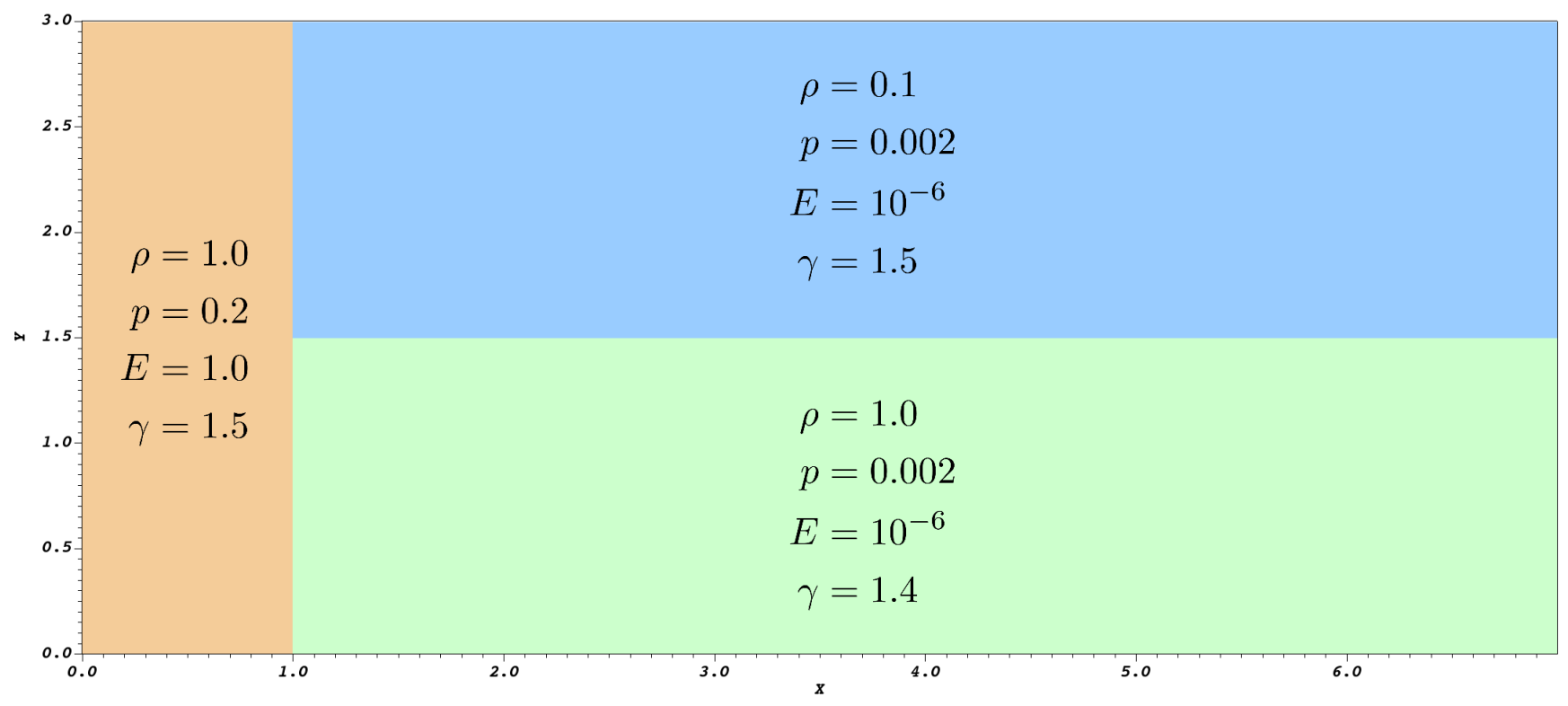

Figure 4: Initial conditions for the triple point problem, including density $\rho$, pressure $p$, radiation energy density $E$, and the ratio of specific heats, $\gamma$. The units are listed in Sec. 4.2.

\begin{tabular}{|c|c|c|c|c|c|c|c|}
\hline Code & Dimension & End time & Points/cells & Procs & Steps & Time per step & Energy error \\
\hline \hline \multirow{2}{*}{ Spheral } & 2 & 1.75 & 328,465 & 288 & 19,264 & 1.814 & $9.286 \times 10^{-4}$ \\
\cline { 2 - 8 } & 3 & 0.7 & $1,058,392$ & 1152 & 3,156 & 25.360 & Not recorded \\
\hline \multirow{2}{*}{ Kull } & 2 & 1.75 & 328,704 & 144 & 63,488 & 0.880 & $-2.416 \times 10^{-3}$ \\
\cline { 2 - 8 } & 3 & 0.7 & 147,980 & 144 & 4,795 & 1.754 & $1.181 \times 10^{-3}$ \\
\hline
\end{tabular}

Table 5: Timing and step information for the ablation problem. The energy error is the initial minus the final energy, divided by the initial energy. 


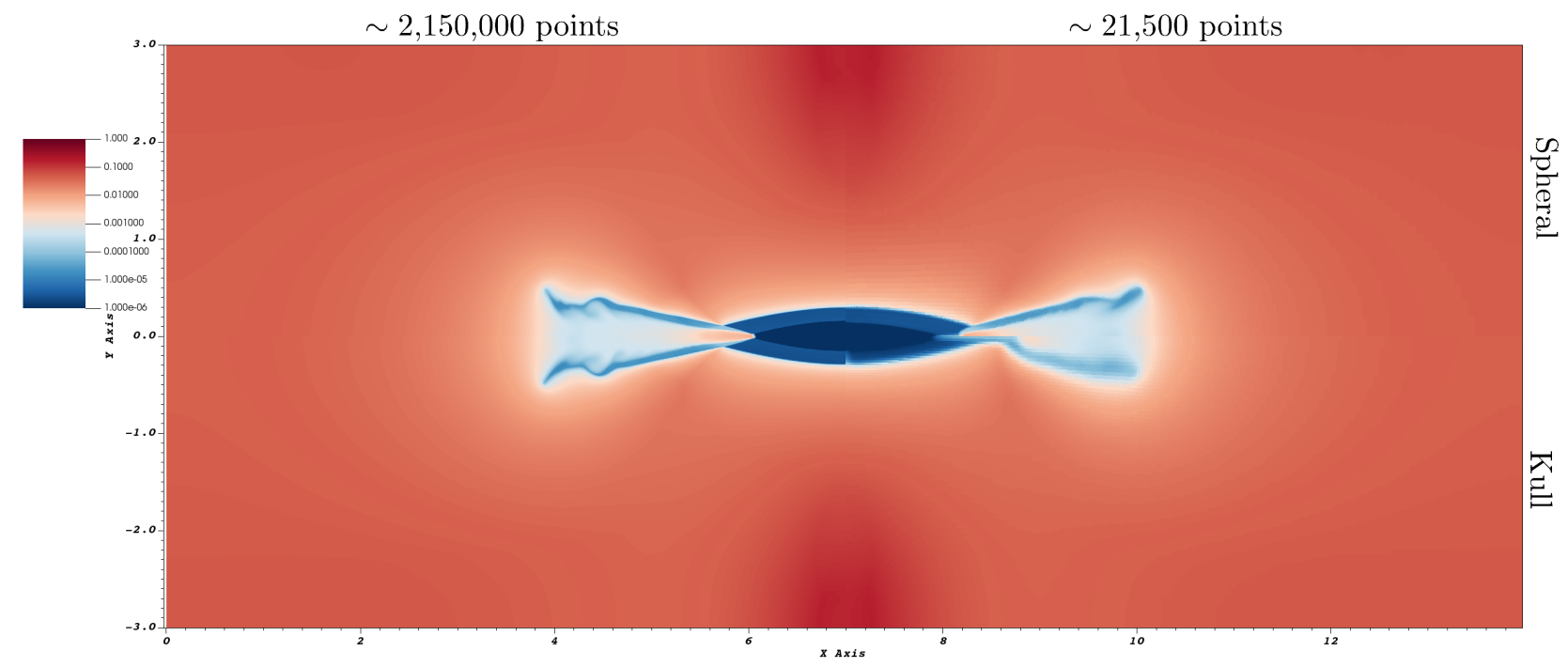

(a) Radiation energy

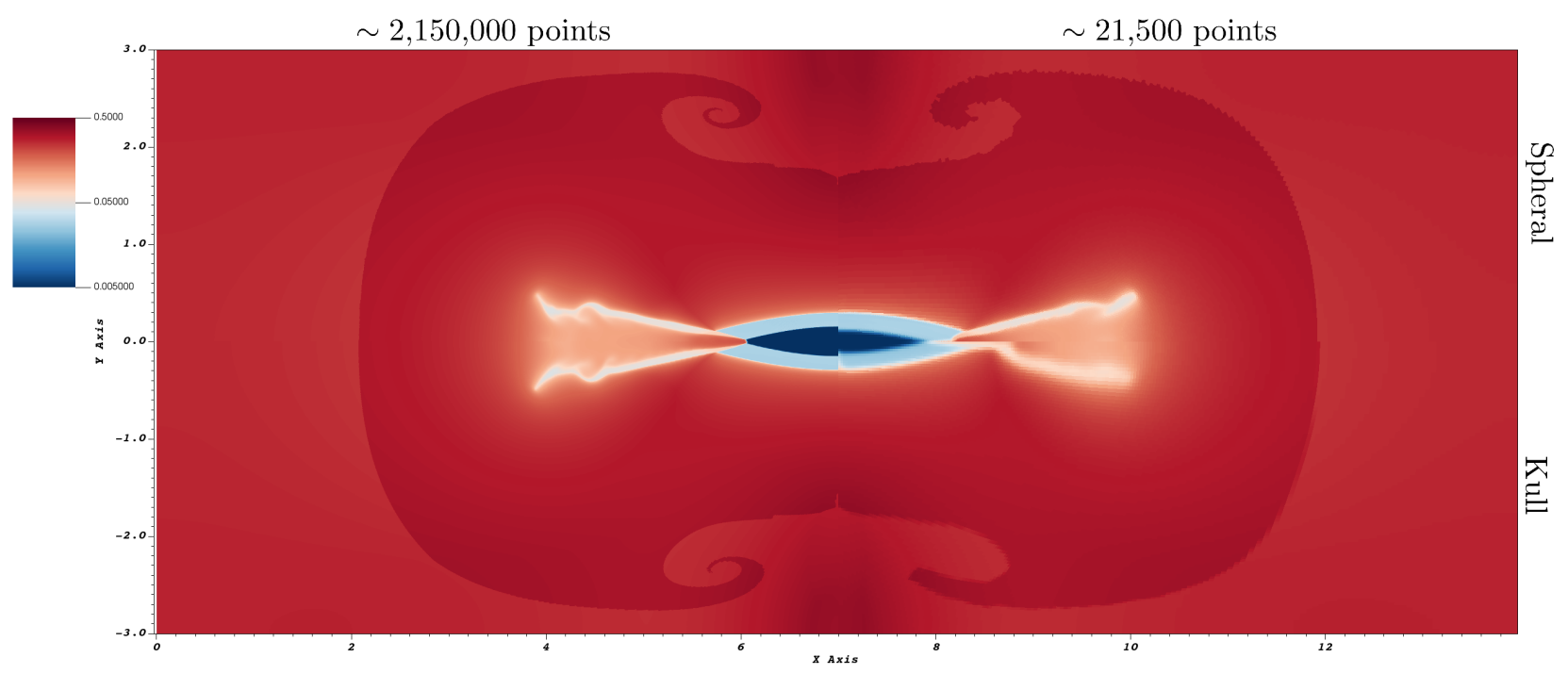

(b) Material energy

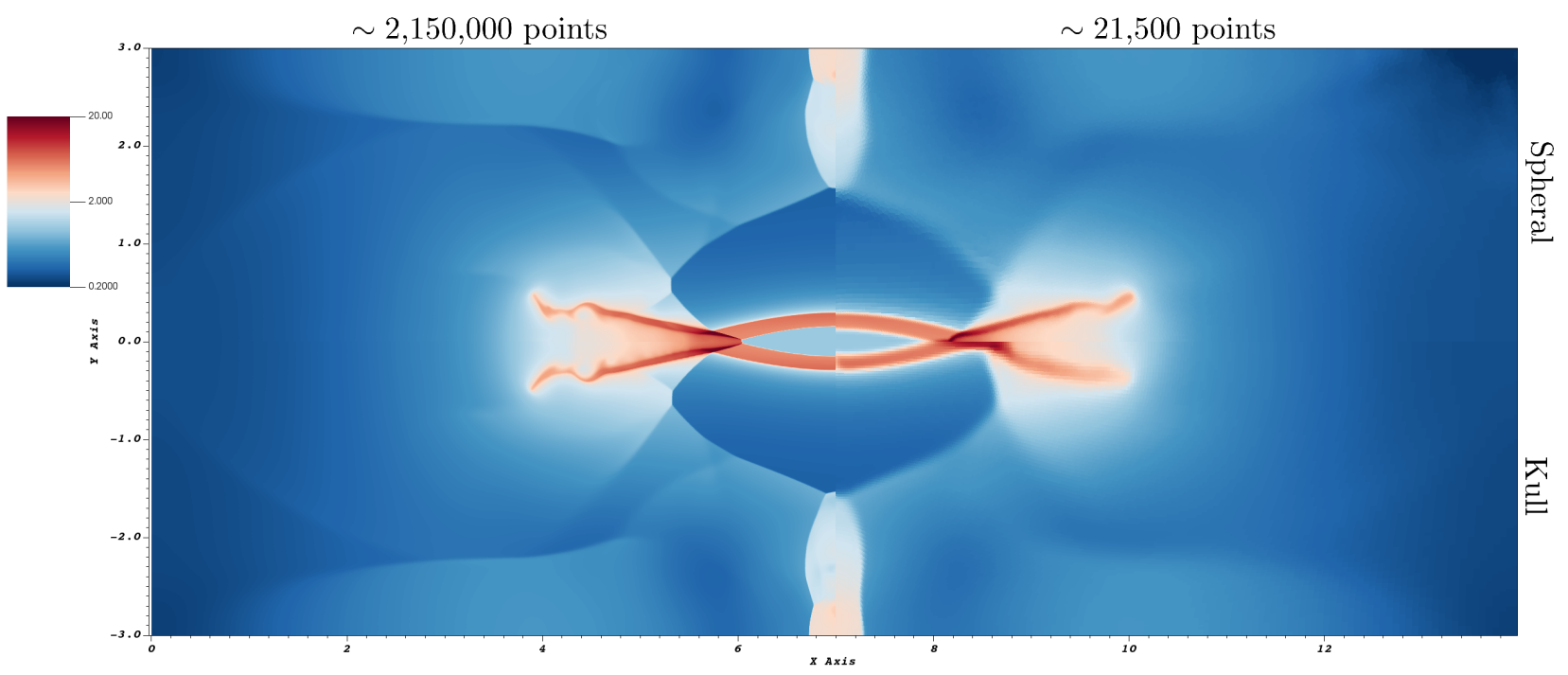

(c) Density

Figure 5: Comparison of four test cases for the triple point problem at $7 \mathrm{sh}$. For each plot, the top half is Spheral and the bottom half is Kull. The left half is the high-resolution version, and the right half is the low-resolution version. This figure is also available as a video. 


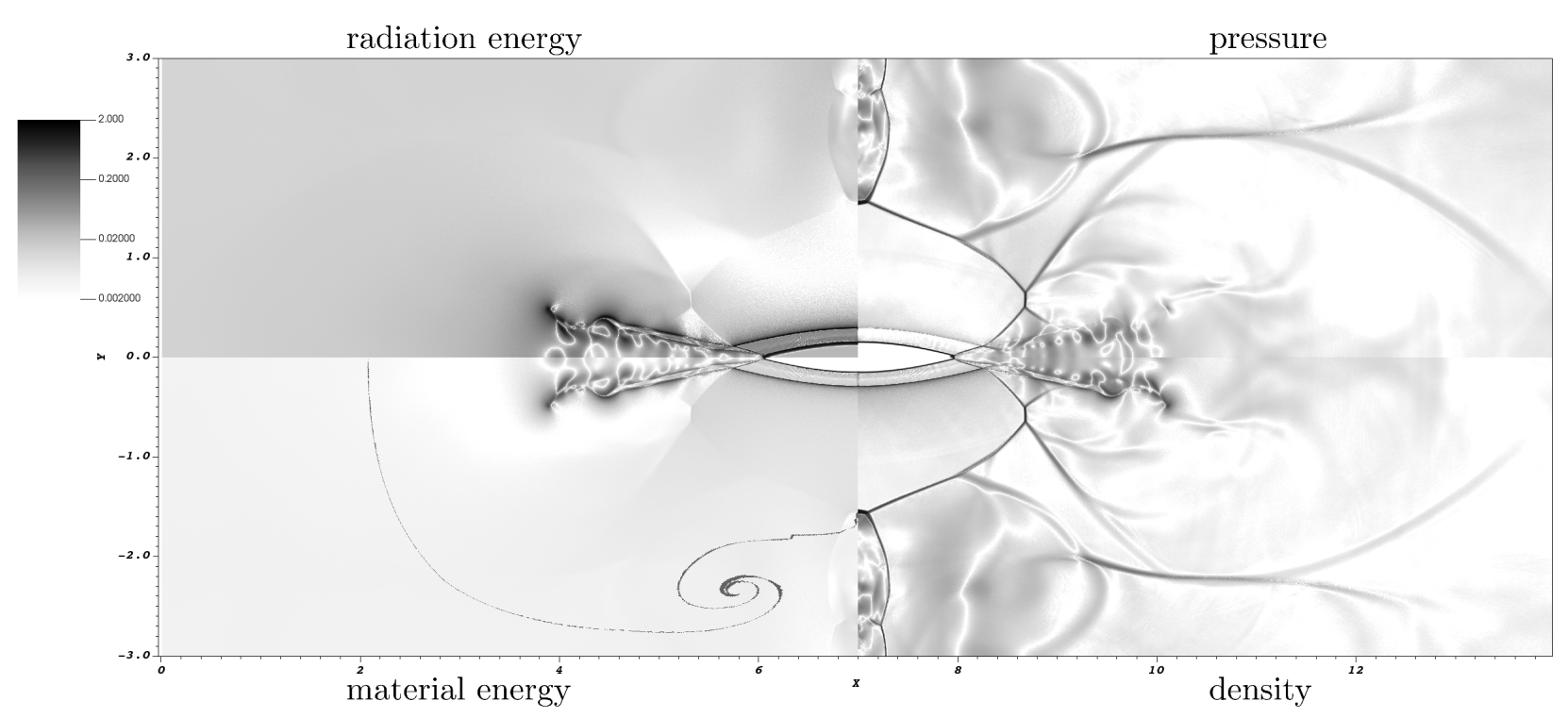

Figure 6: The difference between Spheral and Kull state variables, divided by the average [Eq. (34)], for the triple point problem run with around 2,150,000 points or cells at $7 \mathrm{sh}$. This figure is also available as a video.

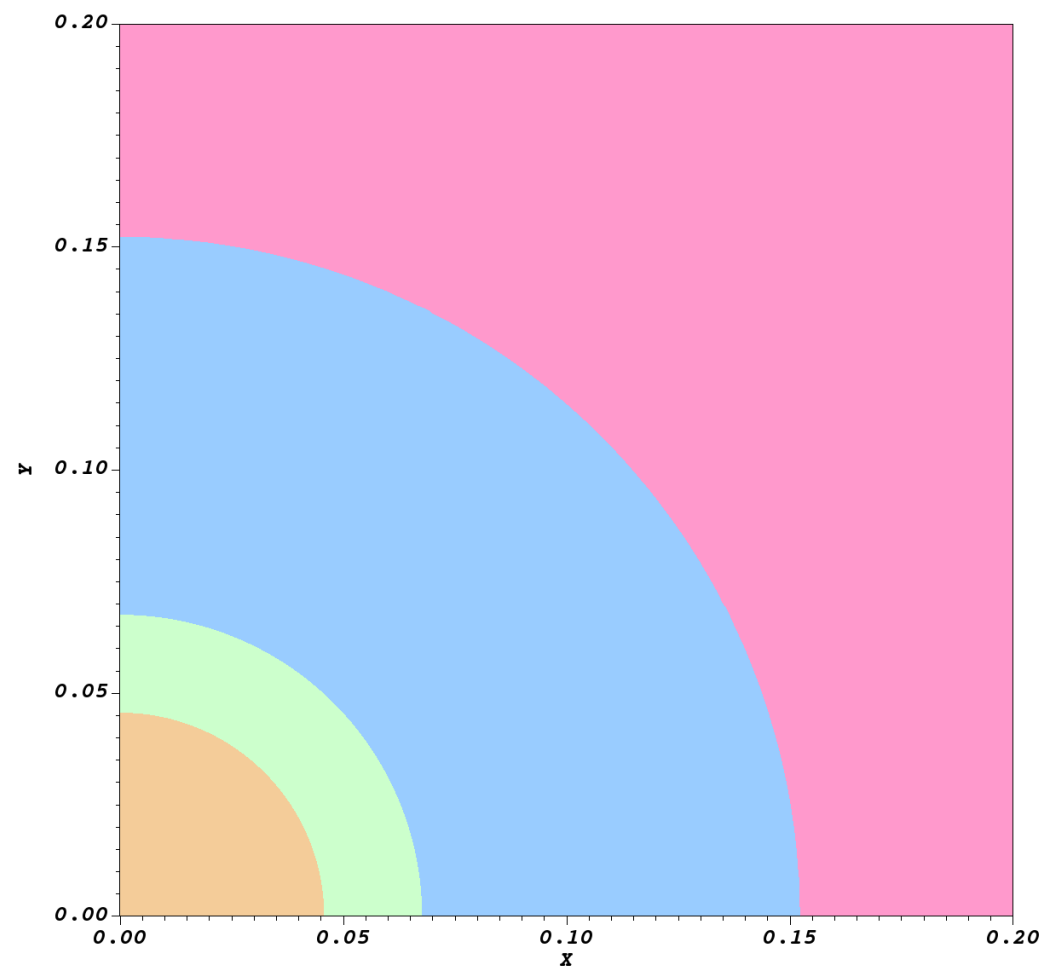

Figure 7: Geometry for the ablation problem. From the origin out, the regions are DT gas, DT ice, CH $1 \% \mathrm{Si}$ plastic, and helium gas. In 2D, the shells are infinite cylinders, while in $3 \mathrm{D}$, the shells are spheres. 


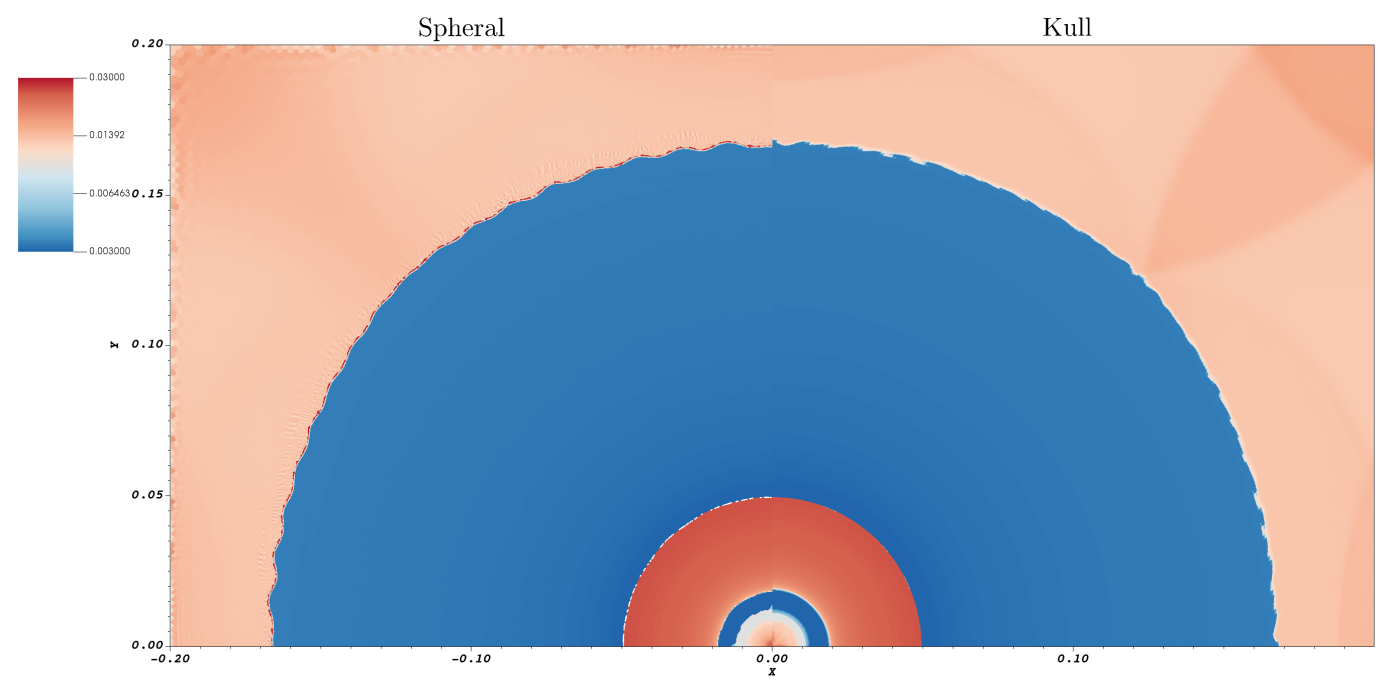

(a) Material energy

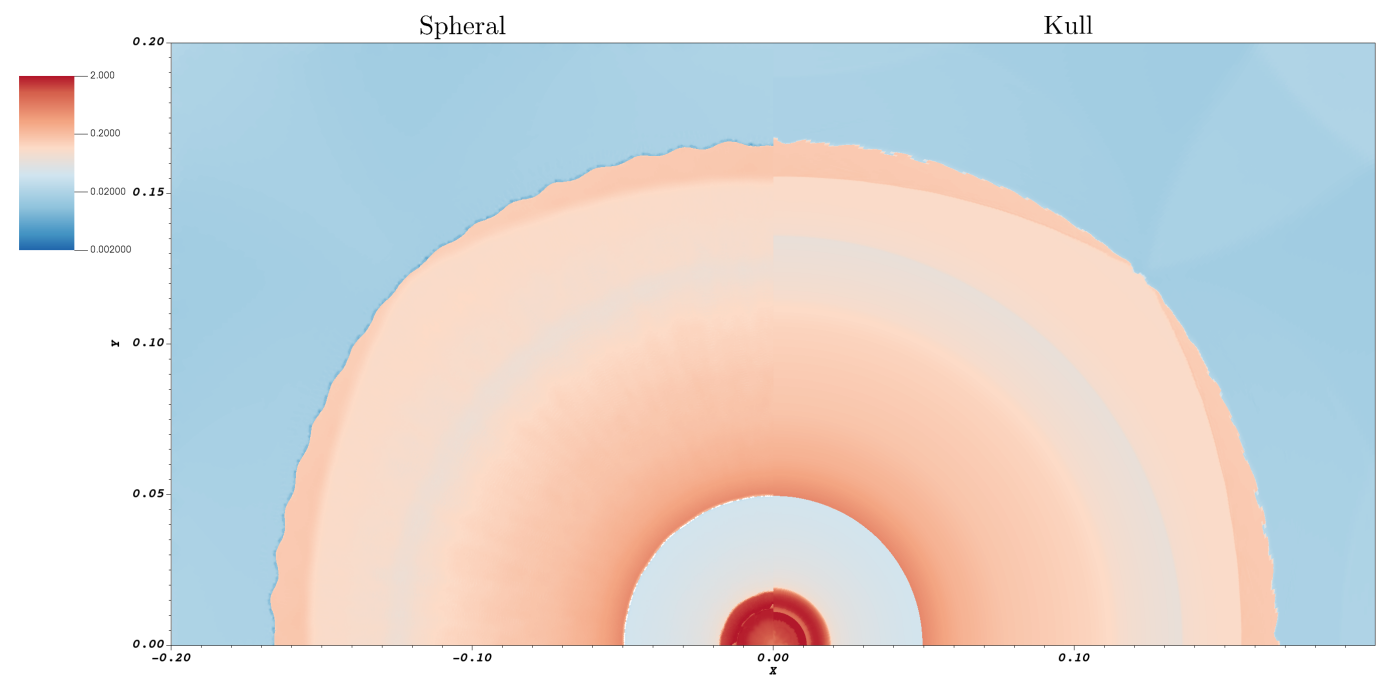

(b) Density

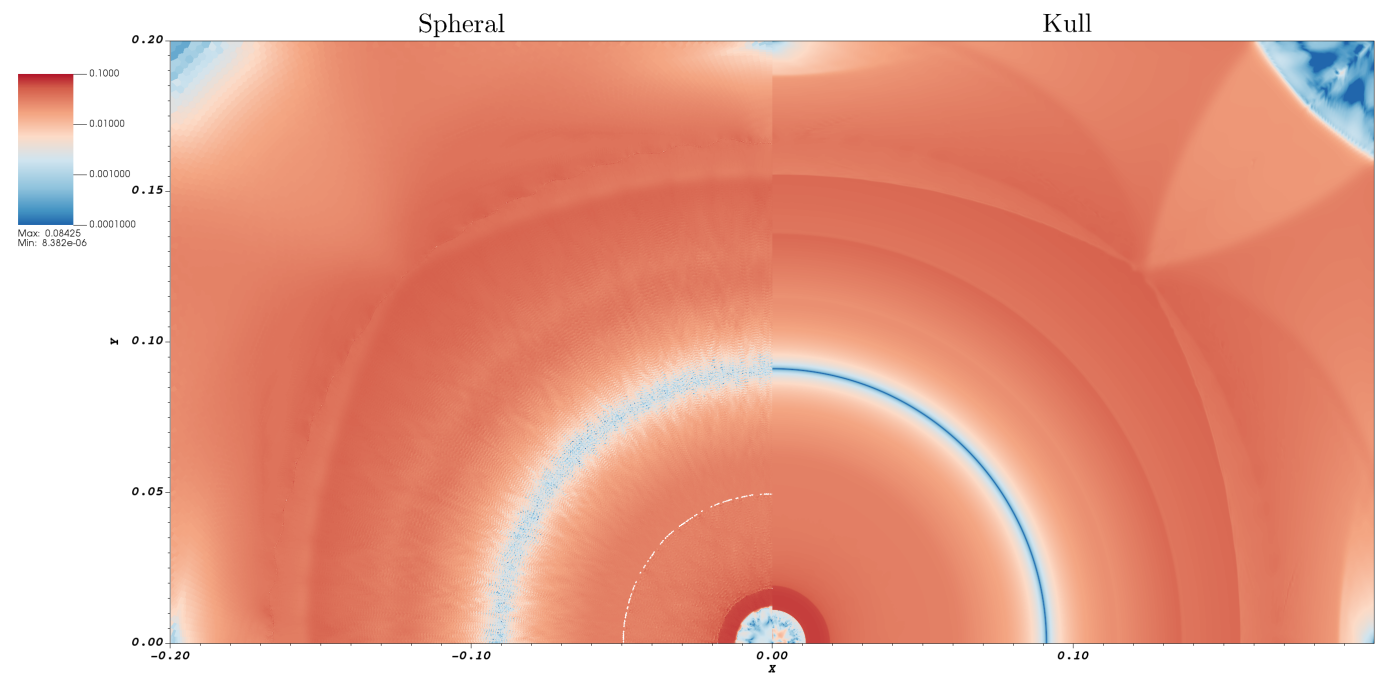

(c) Velocity

Figure 8: Comparison of Kull and Spheral for the ablation problem in 2D at $1.2 \mathrm{sh}$, at maximum compression of the gas. See Table 4 for the spatial discretization information. This figure is also available as a video. 


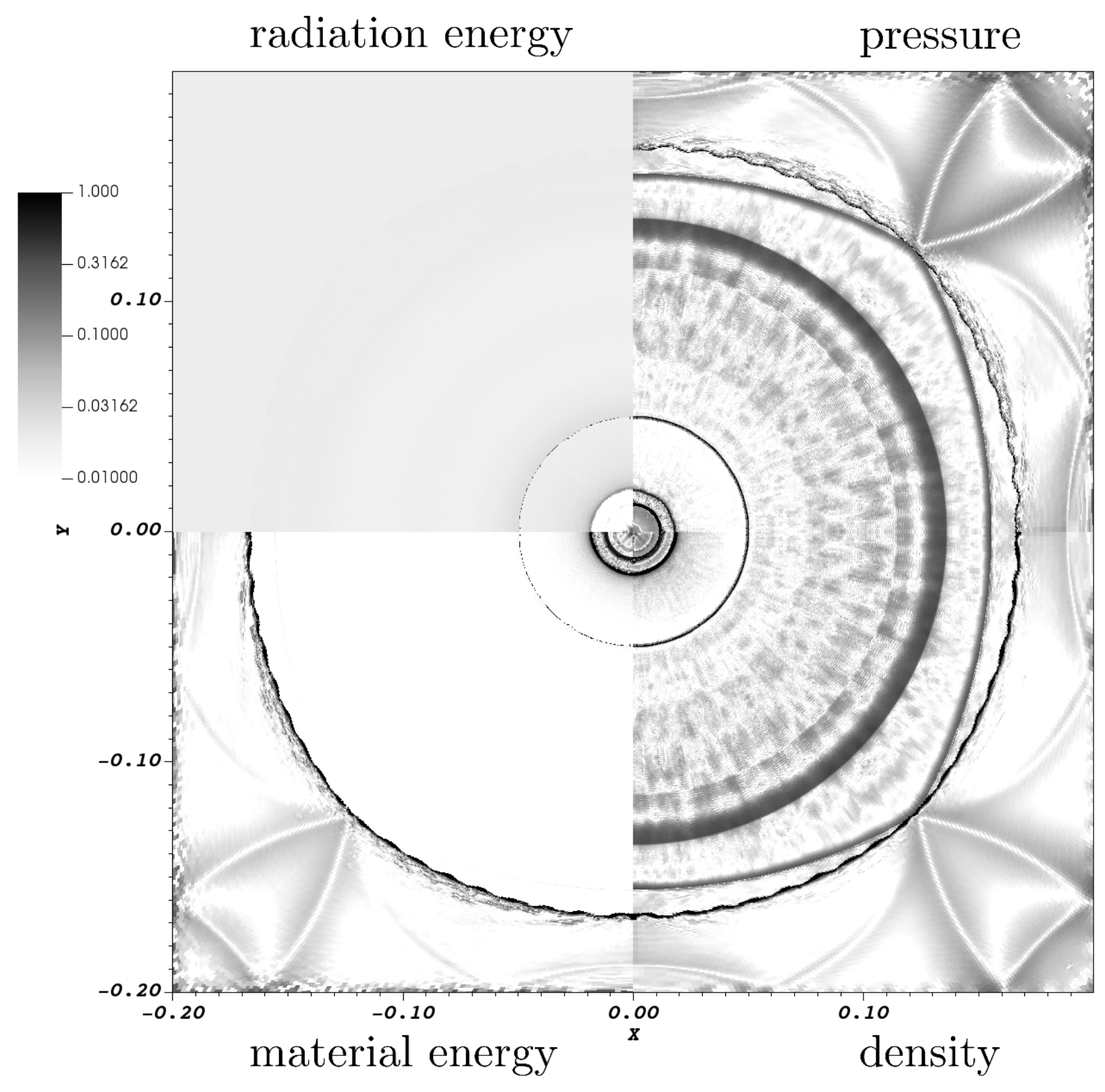

Figure 9: The difference between Spheral and Kull state variables, divided by the average [Eq. (34)], for the ablation problem in $2 \mathrm{D}$ at $1.2 \mathrm{sh}$. This figure is also available as a video. 


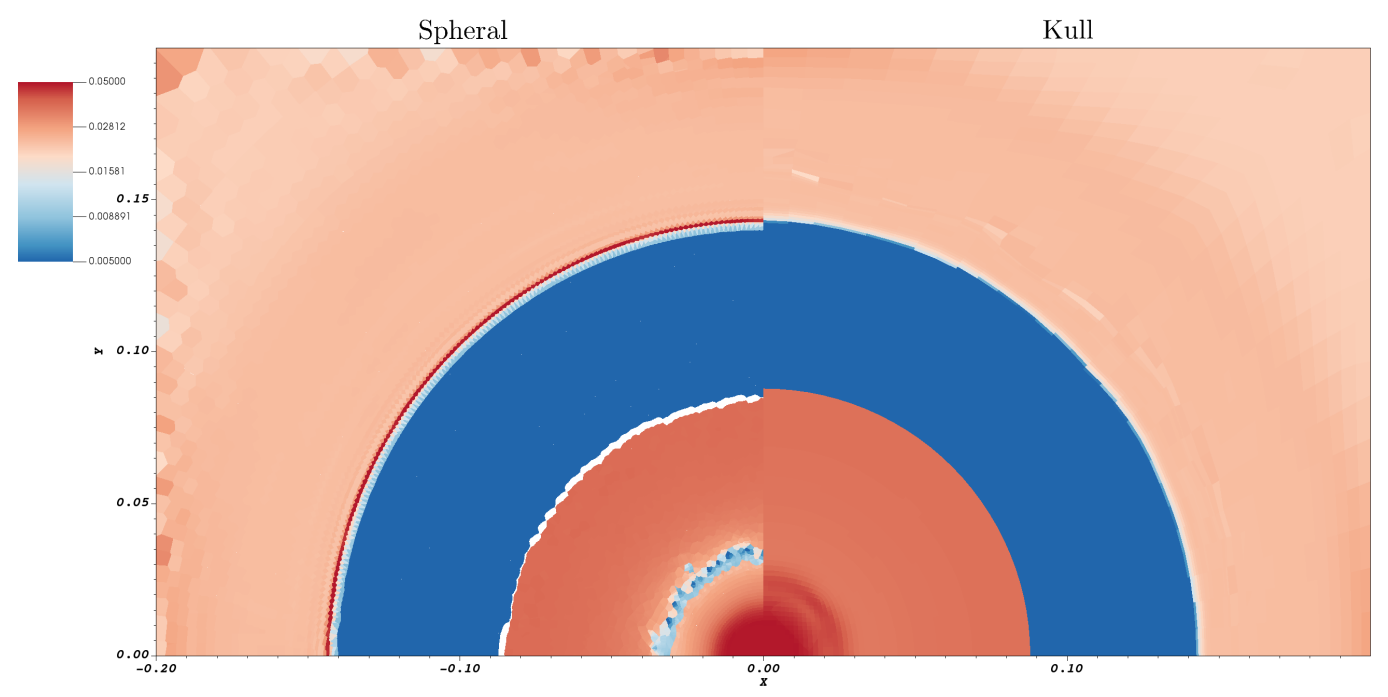

(a) Material energy

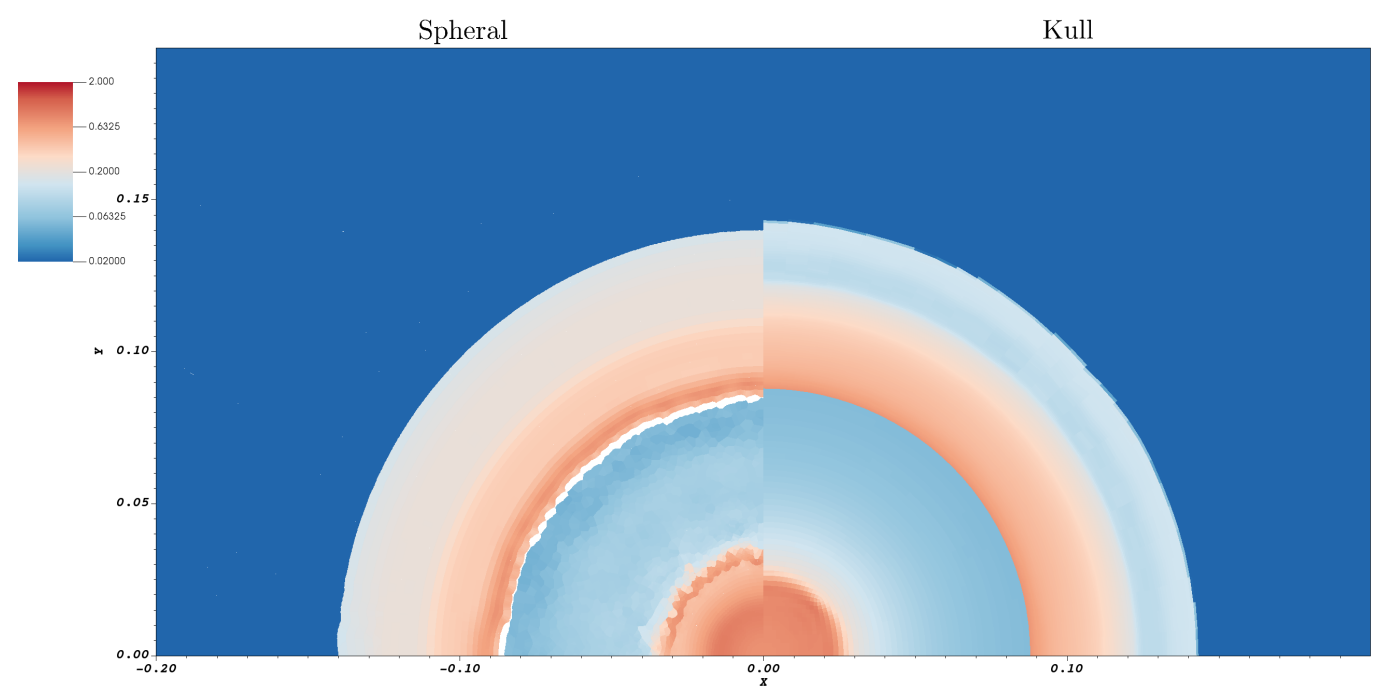

(b) Density

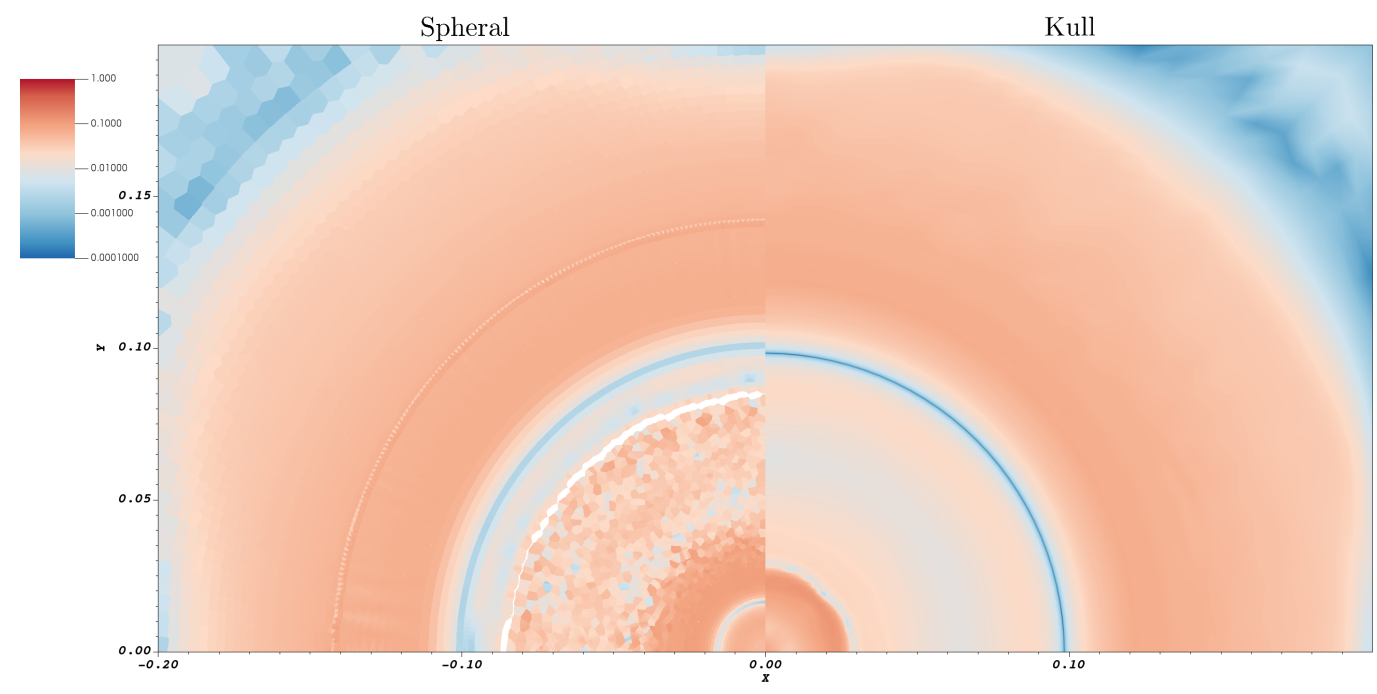

(c) Velocity

Figure 10: Comparison of Kull and Spheral for the ablation problem in 3D at 0.48 sh. See Table 4 for the spatial discretization information. This figure is also available as a video. 